\title{
SUSY: Quo Vadis?
}

\author{
G. G. Ross ${ }^{\mathrm{a}}$ \\ Rudolf Peierls Centre for Theoretical Physics, University of Oxford, 1 Keble Road, Oxford OX1 3NP, UK
}

Received: 27 November 2013 / Accepted: 27 November 2013 / Published online: 27 May 2014

(C) The Author(s) 2014. This article is published with open access at Springerlink.com

\begin{abstract}
Given that there is currently no direct evidence for supersymmetric particles at the LHC it is timely to reevaluate the need for low scale supersymmetry and to ask whether it is likely to be discoverable by the LHC running at its full energy. We review the status of simple SUSY extensions of the Standard Model in the light of the Higgs discovery and the non-observation of evidence for SUSY at the LHC. The need for large radiative corrections to drive the Higgs mass up to $126 \mathrm{GeV}$ and for the coloured SUSY states to be heavy to explain their non-observation introduces a little hierarchy problem and we discuss how to quantify the associated fine tuning. The requirement of low fine tuning requires non-minimal SUSY extensions and we discuss the nature and phenomenology of models which still have perfectly acceptable low fine tuning. A brief discussion of SUSY flavour-changing and CP-violation problems and their resolution is presented.
\end{abstract}

\section{Introduction}

To date, the data from the $\mathrm{LHC}$ running at $8 \mathrm{TeV}$ has shown no indication of supersymmetric partners of the Standard Model states nor, indeed, any indication of other physics 'Beyond the Standard Model' (BSM). Moreover the discovery of a new state whose properties look just like those of the Higgs boson predicted by the Standard Model has led to a reevaluation of the need for such BSM physics. In this paper we will briefly review the reasons why supersymmetry was and still is the most promising extension of the Standard Model (SM) and why the new supersymmetric states are expected to be relatively low in mass and accessible to discovery when the LHC runs at its full energy.

The SM provides an amazingly precise description of the strong, weak and electromagnetic interactions of the fundamental states of matter, the quarks and leptons. However,

a e-mail: g.ross1@physics.ox.ac.uk there are several reasons to think that it is incomplete. The SM does not have a viable candidate for dark matter. The measurement of neutrino masses and the observation of nontrivial mixing in the lepton sector certainly require an extension of the original formulation of the SM-the most elegant possibility is to add right-handed $(\mathrm{RH})$ neutrinos, which restores the symmetry between quarks and leptons and allows for neutrino masses and mixing. It has a large number of unrelated parameters needed to describe the strengths and properties of the fundamental interactions and the masses and mixing angles of the quarks and leptons suggesting there is a more fundamental theory capable of fixing these parameters. Although the Standard Model does provide a measure of unification between the weak and electromagnetic interactions it falls short of a complete unification of the fundamental forces. There is also no mechanism in the original SM for baryogenesis or an explanation of the strong CP problem. Lastly, and most pressing, the Standard Model suffers from the hierarchy problem, namely the difficulty in field theory of separating mass scales so that the electroweak scale is expected to be driven close to any high scale associated with BSM physics, such as the Planck scale or the Grand Unified (GUT) scale. Given the importance of looking for evidence of BSM physics, particularly timely in view of the LHC programme, let us address these reasons in turn.

The need for dark matter is certainly important but does not, by itself, set the scale for the new physics. It could, for example, be due to axions associated with a very high scale of new physics, perhaps even as high as the Planck scale, and evidence for it may not be accessible at laboratory energy scales. Similarly the new physics associated with neutrino masses could be very large. Indeed if the RH neutrinos have mass close to the GUT scale the smallness of the observed (LH) neutrino's mass is naturally explained via the see-saw mechanism.

The possibility of further unification is certainly appealing and there is significant circumstantial evidence in favour 
of it. In particular it is notable that the SM representations of the quarks and leptons of a single family fit neatly in the relatively simple $\overline{5}+10$ representation of a $\mathrm{SU}(5)$ GUT. This immediately explains the fact that the charges of the quarks are third integrally quantised relative to the charged leptons, the $1 / 3$ factor coming from the fact that quarks come in three colours. Moreover the underlying SU(5) symmetry explains why both the quark and the lepton electroweak SU(2) doublets are left-handed. The resulting simplicity is further enhanced if the GUT group is enlarged to $\mathrm{SO}(10)$ for all the states of a single family fit into a single 16 dimensional representation of $\mathrm{SO}(10)$. Moreover the remaining state needed to complete the 16 dimensional representation requires the existence of a right-handed neutrino and thus naturally allows for neutrino masses and mixing. Of course it is important that the Higgs should also fit into a GUT multiplet and, in the original simple formulations, this led to a problem because the coloured Higgs partners required by the GUT have not been observed. Indeed they are required to be super-heavy or absent if nucleon decay is to be sufficiently suppressed and this led to the need for very large GUT representations that somewhat reduced the elegance of the GUT paradigm. However, the more recent approaches based on an underlying string unification have provided an elegant explanation for the absence of light coloured partners as they can be projected out at the stage of compactification and the associated breaking of the gauge group by Wilson lines, without the need for large GUT representations.

The existence of an underlying GUT also leads to relations amongst the parameters of the SM, most importantly the $\mathrm{SU}(3) \times \mathrm{SU}(2) \times \mathrm{U}(1)$ gauge couplings. In $\mathrm{SU}(5)$ or $\mathrm{SO}(10)$ there is a single gauge coupling and, using the renormalisation group (RG) equations, it is straightforward to determine the strong, electromagnetic and weak couplings at low scales, as measured in the laboratory, in terms of the unified gauge coupling and the unification scale. Eliminating these unknowns leads to a relation between the gauge couplings at low energies. In the non-supersymmetric case this relation fails by more than 11 standard deviations but, in a supersymmetric GUT, the relation is accurate to better than $5 \%$ for the case of light ( $\mathrm{TeV}$ scale) supersymmetric (SUSY) partners. However, as the dependence of this relation on the SUSY masses is only logarithmic, it is not possible to say that gauge coupling unification requires that the SUSY states should be within the reach of the LHC.

This leaves the need to solve the hierarchy problem as the only unambiguous reason to expect new states at a mass scale accessible to direct discovery at the LHC. The problem arises if the SM is an effective field theory descending from new physics at a high scale, such as the GUT scale or the string or Planck scale. In this case the radiative corrections that arise in field theory connect the low and high scale sectors and prevent a separation of the electroweak breaking scale from the high scale associated with BSM physics. Support for the idea that the SM is an effective field theory (EFT) comes from the fact that it predicts that the only elementary spin-one states in the theory should be associated with a local gauge principle and that the only fermions in the theory should be chiral. This immediately follows because the only symmetry capable of forbidding a vector boson mass is a local gauge symmetry and the only symmetry capable of forbidding a fermion mass is a chiral symmetry; in the absence of such symmetries one would expect the states to have the high scale mass associated with the new scale of physics. It is significant that this is just what is found in the SM. The gluons, the photon and the $\mathrm{W}$ and $\mathrm{Z}$ bosons are the gauge bosons coming from the $\mathrm{SU}(3) \times \mathrm{SU}(2) \times \mathrm{U}(1)$ local gauge symmetry. The fermions are chiral with respect to the SU(2) gauge group, the LH fermions transform as SU(2) doublets and the RH fermions transform as SU(2) singlets. Moreover the EFT description requires that the unbroken gauge interactions should be vectorlike, as observed, otherwise there can be no fermion masses.

This pleasing agreement of the SM structure with that required by an EFT is broken by the scalar sector because there is no symmetry that forbids a scalar mass. As a result the EFT expectation is that the Higgs mass and the associated electroweak breaking scale should be at the high scale. Even if for some reason the Higgs mass does not arise at tree level radiative corrections are expected to generate it close to the high scale. This is the hierarchy problem. The solutions that have been suggested require new physics at a low scale, potentially accessible to discovery at the LHC. In this review I will concentrate on the supersymmetric possibility in which the Higgs mass is forbidden by a combination of supersymmetry (SUSY) and a chiral symmetry. In this case the SUSY extension of the SM fits in perfectly with the EFT picture. It also allows for a consistent underlying unified theory, GUT or string based, that gives the precision prediction relating the gauge couplings and string-based models can even predict the correct unification scale in therms of the string or Planck scale. Indeed, due to the inevitable coupling between the Higgs and the scalars responsible for GUT breaking at a high scale, the SUSY extension of the GUT is an essential feature of unification.

The discovery of a Higgs candidate at $126 \mathrm{GeV}[1,2]$ has lent some support to the SUSY paradigm but at the same time made it more difficult to realise. The support comes because the Higgs mass lies in the relatively small range favoured by SUSY. Moreover the observed properties of the candidate Higgs are just what are expected of an elementary state whose interactions are in the perturbative domain, as is expected in SUSY with gauge coupling unification. Thus at least the simplest composite Higgs explanation of the hierarchy problem looks less likely than the SUSY explanation. The fact that the Higgs looks very much like the SM Higgs 
is not an argument against SUSY for, in the limit the SUSY breaking is large, the properties of the SUSY Higgs very closely approximate those of the SM Higgs. The difficulty comes from the relatively high mass of the Higgs that typically requires significant fine tuning in SUSY models due to the need of large radiative corrections to the Higgs mass and the corresponding need for heavy SUSY states. This fact largely maps out the possible nature of SUSY extensions of the SM after the Higgs discovery for it limits the available parameter space and structure of SUSY models and may indicate the need for a non-minimal SUSY extension of the SM. In this paper we consider these possibilities in more detail and the prospects for SUSY discovery at LHC14.

The structure of the paper is as follows. In Sect. 2 the status of simple SUSY models after the Higgs discovery is reviewed and their implications for the masses of the coloured SUSY states, particularly relevant to the LHC. In Sect. 3 we discuss the hierarchy problem and the quantitative 'fine tuning' measure of the hierarchy problem that is needed to obtain a prediction for the SUSY partner mass scale and the prospects for finding SUSY at the LHC. In Sect. 4 we review the applications of the measure to SUSY extensions of the SM and discuss whether there remain viable SUSY theories capable of solving the 'little' hierarchy problem, the particular SUSY signals implied by them and the prospects for testing them at the LHC. In Sect. 5 we discuss SUSY implications for flavour physics in the light of the constraints the LHC results have imposed on SUSY, concentrating on the most sensitive flavour-changing and $\mathrm{CP}$-violating processes. Finally in Sect. 6 we present a summary and our conclusions.

\section{Status of simple SUSY models after the Higgs}

In this section we briefly review the status of fits to data of the simplest and most studied of the SUSY extensions of the SM, taking account of the Higgs discovery, the limits on SUSY states coming from the LHC and other experiments and the constraints imposed by the observed dark matter abundance.

In such models the Higgs mass is strongly constrained. At tree level it is bounded by the $Z$ mass but there can be sizeable radiative contributions increasing it. However, these contributions cannot be made arbitrarily large unless the SUSY breaking scale is taken so high that unacceptable fine tuning is needed to generate the EW breaking scale. The $125 \mathrm{GeV}$ Higgs lies at the upper end of this range. On the one hand it may be considered a success for the SUSY prediction of a light Higgs with couplings in the perturbative domain. On the other hand it is sufficiently heavy for considerable fine tuning of the fundamental parameters of the model to be needed, at least in the minimal implementations of SUSY.
In the next section we will quantify this fine tuning but in this section we choose to ignore it, assuming that the underlying theory fixes the necessary correlations between the parameters needed to get the correct EW breaking scale or that higher dimension operator contributions due to new states at a higher scale are present (cf. the discussion in Sect. 4.4). The spectrum of the simplest models is that of the MSSM with the minimal set of chiral super fields, $\phi^{i}$, needed to accommodate the matter fields of the SM plus the two Higgs doublets needed to give mass to the up and down quarks. The scalar potential then has the form

$V\left(\phi^{i}\right)=\left|\frac{\partial W}{\partial \phi^{i}}\right|^{2}+\left(A_{0} W^{(3)}+B_{0} W^{(2)}+\right.$ h.c. $)+m_{0}^{2} \phi^{i} \phi_{i}^{*}$,

where $W$ is the superpotential for the matter fields given by

$$
\begin{aligned}
& W_{\text {MSSM }}=W_{\text {Yukawa }}+W_{\mu} \\
& W_{\text {Yukawa }}=y_{e} H_{d} L e^{c}+y_{d} H_{d} Q d^{c}+y_{u} H_{u} Q u^{c} \\
& W_{\mu}=\mu H_{u} H_{d},
\end{aligned}
$$

The form of the SUSY breaking terms assumed here is that of the constrained MSSM (CMSSM) with scalar mass universality, applicable at some initial scale, $M_{X}$, which is usually associated with the Grand Unification scale. In these equations the family and $\mathrm{SU}(2)$ indices have been suppressed, $W^{(3)}$ is the trilinear part of the superpotential and $W^{(2)}$ is the bilinear part. Gaugino mass universality characterised by $m_{1 / 2}$ is also assumed at the same input scale $M_{X}$.

Minimisation of the Higgs potential leads to two vacuum conditions at the weak scale, which can be expressed as

$\mu^{2}=\frac{m_{1}^{2}-m_{2}^{2} \tan ^{2} \beta+\frac{1}{2} m_{Z}^{2}\left(1-\tan ^{2} \beta\right)+\Delta_{\mu}^{(1)}}{\tan ^{2} \beta-1+\Delta_{\mu}^{(2)}}$

and

$B \mu=-\frac{1}{2}\left(m_{1}^{2}+m_{2}^{2}+2 \mu^{2}\right) \sin 2 \beta+\Delta_{B}$,

where $m_{1,2}$ are the soft supersymmetry-breaking Higgs masses (evaluated at the weak scale), $\tan \beta$ is the ratio of the two Higgs vacuum expectation values, and $\Delta_{B}$ and $\Delta_{\mu}^{(1,2)}$ are loop corrections [3-5]. Using these relations we see that the CMSSM can be defined by $m_{1 / 2}, m_{0}, A_{0}, \tan \beta$ and the sign of $\mu$.

The Higgs mass is given by [6-8]

$m_{h}^{2} \simeq m_{Z}^{2} \cos ^{2} 2 \beta+\frac{3}{(4 \pi)^{2}}\left[\ln \frac{M_{S}^{2}}{m_{t}^{2}}+\frac{X_{t}^{2}}{m_{\tilde{t}}^{2}}\left(1-\frac{X_{t}^{2}}{12 m_{\tilde{t}}^{2}}\right)\right]$

where $M_{S}^{2}=m_{\tilde{t}_{L}} m_{\tilde{t}_{R}}, X_{t}=A_{0}-\mu \cot \beta$ and the parameters are evaluated at the EW scale. From this one may see that to get large radiative corrections requires a large stop mass 

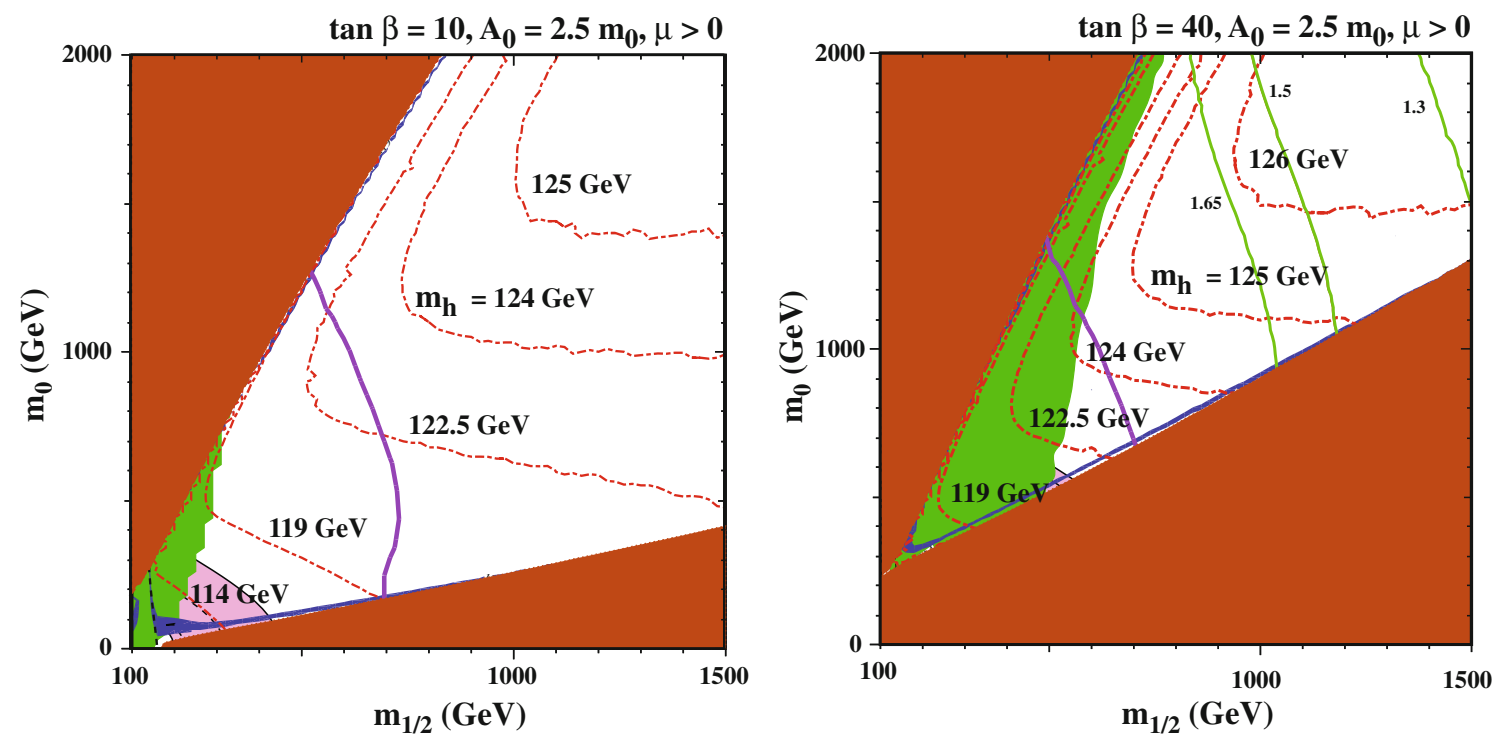

Fig. 1 The CMSSM $\left(m_{1 / 2}, m_{0}\right)$ planes for $\mu>0$, with $\tan \beta=10$ (left) and 40 (right), $A_{0}=2.5 m_{0}$, as calculated for $m_{t}=173.2 \mathrm{GeV}$ using the latest version of the SSARD code [79]. The interpretations of the shadings and contour colours are described in the text

and/or $X_{t}$, leading to very heavy coloured SUSY states in the $\mathrm{TeV}$ range.

\subsection{CMSSM fits}

Several groups [9-59] have performed detailed CMSSM fits to the LHC data from Atlas and CMS $[60,61]$. In these models the lightest SUSY particle (LSP), usually a combination of a neutralino and a Higgsino, is stable and a dark matter (DM) candidate. The fits that we discuss require that the DM should saturate at or be below the preferred cosmological range $\Omega_{\mathrm{CDM}} h^{2}=0.112 \pm 0.006$ [62], where $h$ is the present Hubble expansion rate in units of $100 \mathrm{~km} / \mathrm{s} / \mathrm{Mpc}$. In addition the dark matter should be consistent with the direct detection bounds, the strongest in the mass range of interest being that of XENON100 [63]. Constraints are also provided by flavour physics, especially $b \rightarrow s \gamma$ [64-67] and $B_{s} \rightarrow \mu^{+} \mu^{-}$[68-73]. The LHCb measurement of the latter with a value close to the SM prediction provides particularly strong constraints on the large $\tan \beta$ region. In addition the measured value $[74,75]$ of the anomalous magnetic moment of the muon, $g_{\mu}-2$, plays a significant role in the fits. We will discuss further aspects of flavour-changing and $\mathrm{CP}$-violation constraints in Sect. 5.

In Fig. 1 we show the results of a recent fit [54] to all the available data, including the latest $\mathrm{LHCb}$ measurement of $B_{s} \rightarrow \mu^{+} \mu^{-}$, for the parameters $m_{0}$ and $m_{1 / 2}$ as a function of the Higgs mass contours (red dashed lines). In each panel, the region at high $m_{1 / 2}$ and low $m_{0}$ where the $\tilde{\tau}_{1}$ is the LSP is shaded brown, as is the region at low $m_{1 / 2}$ and high $m_{0}$ where the stop becomes the LSP (or tachyonic). The regions excluded by $b \rightarrow s \gamma$ are shaded green, those favoured by $g_{\mu}-2$ are shaded pink, and those favoured by $\Omega_{\chi} h^{2}$ are shaded dark blue ${ }^{1}$. The LEP chargino exclusion is shown as a near-vertical dashed black line at small $m_{1 / 2}$ [76]. The ATLAS exclusion from the absence of missing transverse energy events (MET) is shown by the purple lines. Also shown as solid green lines are three contours of $\mathrm{BR}\left(B_{s} \rightarrow \mu^{+} \mu^{-}\right) / \mathrm{BR}\left(B_{s} \rightarrow \mu^{+} \mu^{-}\right)_{\mathrm{SM}}=1.65,1.5$ (the present $95 \%$ CL upper limit from LHCb and combined experiments), and 1.3 (the $68 \%$ upper limit from LHCb).

Over the range shown in the $\left(m_{0}, m_{1 / 2}\right)$ plane a large value for $A_{0}$ is needed to get to the observed Higgs mass ${ }^{2}$. For larger values of $\tan \beta$ the Higgs mass increases but the $B_{s} \rightarrow \mu^{+} \mu^{-}$ rate still requires that $m_{0}$ and $m_{1 / 2}$ are above $1 \mathrm{TeV}$. However, taking account of the dark matter constraint, for $\tan \beta=10$, $M_{h}$ does not grow above $\sim 121 \mathrm{GeV}$, whereas for $\tan \beta=$ 40 there is compatibility for $m_{1 / 2} \geq 1 \mathrm{TeV}$ along the stau co-annihilation strip (SC) close to the stau LSP boundary ${ }^{3}$. This region is also compatible with the LHC MET constraint, but not with the supersymmetric interpretation of $g_{\mu}-2$. Interestingly the sensitivity of upcoming direct DM searches will be sufficient to test the most likely regions of the CMSSM DM space providing complementary tests to the LHC SUSY searches. LUX will be sensitive to a substantial proportion of the favoured regions and XENON1T will cover almost

\footnotetext{
${ }^{1}$ For reasons of visibility the wider strips $0.06<\Omega_{\chi} h^{2}<0.2$ are shown here.

${ }^{2} M_{h}=125.7 \pm 1.0 \mathrm{GeV}[1,2]$ although, including theoretical errors, values greater than $122 \mathrm{GeV}$ are probably acceptable.

3 The focus point (FP) region, in which the DM unpolarised annihilation cross section is enhanced by a significant Higgsino component of the LSP, is excluded by the XENON100 bound.
} 
all of the rest. This complementarity is important because the majority of points have a very heavy Higgsino LSP, of $O(1 \mathrm{TeV})$, possibly beyond the reach of LHC14.

In the absence of the fine tuning constraint the upper bound on the SUSY mass spectrum comes from the requirement that the SUSY LSP should provide the observed dark matter abundance. For example, a recent Baysian fit [77] finds $m_{\tilde{g}}=3.4 \mathrm{TeV}, m_{\tilde{t}}=2.8 \mathrm{TeV}, m_{\tilde{u}_{L}}=3.5 \mathrm{TeV}$ and $m_{\chi}=700 \mathrm{GeV}^{4}$ as the most probable values suggesting that even LHC14 may not discover evidence for SUSY.

\subsection{Beyond the CMSSM}

As we have seen, the LHC and dark matter constraints have forced the CMSSM parameter space into a tight corner that requires large values of $\tan \beta, A_{0}$ and $m_{1 / 2}$ and a correspondingly heavy SUSY spectrum. However, by extending the parameters of the model the fit constraints can be more readily satisfied, particularly that of obtaining acceptable dark matter abundance. One possibility is to allow the Higgs masses to differ from the squark and slepton masses at the initial scale $[16,18,80-93]$ and in this case it is not difficult to find regions of the parameter space where the cosmological cold dark matter density falls within the preferred range, even if the sparticle masses are relatively large, as required by the LHC MET and $M_{h}$ constraints. For example [54], this may happen in a transition region where the $\chi$ LSP has a relatively large Higgsino component, a region disfavoured in the CMSSM by the XENON100 upper limit on cold dark matter scattering. Another possibility is to lower the initial scale, $M_{X}$, which compresses the spectrum making more co-annihilation processes important, thus suppressing the relic density below the range expected in the CMSSM. While these generalisations of the CNMSSM open up the phase space of acceptable solutions they still require large values of the SUSY breaking parameters corresponding to heavy SUSY spectra similar to those found in the CMSSM.

\subsubsection{The CNMSSM}

Another possibility that has been widely studied is to increase the particle content, for example by adding a gauge singlet chiral super field, $S$. The simplest version of this, the NMSSM (for reviews see $[94,95]$ ), has the superpotential

$W_{\mathrm{NMSSM}}=\lambda S H_{u} H_{d}+\frac{\kappa}{3} S^{3}+W_{\text {Yukawa }}$,

where additional terms are forbidden by the $Z_{3}$ symmetry of $W_{\text {NMSSM }}$. This lacks the bare $\mu$ term of the CMSSM, Eq. (2), but an effective term, $\mu_{\text {eff }}$, is generated at the stage of EW breaking when the scalar component of $S$ acquires a

$\overline{4}$ These values are close to the favoured upper values in [78]. vacuum expectation value (VEV). The advantage of this is that $\mu_{\text {eff }}$ is automatically of the order of the EW breaking scale in comparison to the MSSM case in which the symmetries allow an arbitrarily large $\mu$ term. In the constrained version of the model (CNMSSM) the soft SUSY breaking terms associated with the Higgs sector at the initial scale have the form

$$
\begin{aligned}
V_{\text {soft }}= & m_{0}^{2}\left|H_{u}\right|^{2}+m_{0}^{2}\left|H_{d}\right|^{2}+m_{S}^{2}|S|^{2} \\
& +\left(\lambda A_{0} S H_{u} H_{d}+\frac{1}{3} \kappa A_{0} S^{3}+\text { h.c. }\right),
\end{aligned}
$$

with scalar mass universality assumed for all scalars apart from the new singlet. In this case the parameters of the CNMSSM may be chosen as $m_{0}, m_{1 / 2}, A_{0}, \tan \beta, \lambda, m_{S}$ and the sign of $\mu_{\text {eff }}$.

Several groups have analysed the NMSSM after the Higgs discovery [96-118]. Here we present results from a very recent study [118] that takes account of the measurement of $B_{s} \rightarrow \mu^{+} \mu^{-}$. In the upper figures of Fig. 2 we present the results of a CNMSSM Baysian fit showing $\left(m_{o}, m_{1 / 2}\right)$ planes for $\mu_{\text {eff }}>0$. Because of the additional singlet scalar state there are two CP even Higgs states involving mixtures of the EW doublet and singlet states. Two cases are shown, case 1 in which the lightest CP even Higgs has mass $m_{h_{1}}=125.8 \mathrm{GeV}$ and case 2 in which the second lightest $\mathrm{CP}$ even Higgs has mass $m_{h_{2}}=125.8 \mathrm{GeV}$. Note that in the second of the top figures the right-hand favoured region is already excluded by XENON100 so in both cases both the squarks and the gluinos are in the $\mathrm{TeV}$ range with the gluinos typically heavier than the squarks.

In the lower figure of Fig. 2 we show the $\left(m_{h_{1}}, m_{h_{2}}\right)$ planes for these two cases. One may see from the first figure that the second state, which is mainly singlet, is expected to be very heavy, in the $\mathrm{TeV}$ range, while in the second figure the mainly singlet state can be relatively light but, because of its small doublet component, would not have been detected at LEP. Not shown here is an interesting third case in which both Higgs states have mass in the $126 \mathrm{GeV}$ region.

The fit to the model shows that in case 1 there are two viable DM regions, one, the A-funnel region in which neutralinos annihilate through the resonance with the lightest pseudoscalar and a second (SC) in which the dominant annihilation process is through stau-coannilation. In the upper left figure the $\mathrm{SC}$ region is that including the best fit point, while the $\mathrm{AF}$ region corresponds to the top part of the plot. Both regions lie below the XENON100 limits but will be partly probed by XENON1T. In case 2 the best fit region is again $\mathrm{SC}$ and its interaction cross section lies below even the sensitivity of XENON1T. However, the other region corresponds to the focus point region and is already inconsistent with the XENON100 bound. 

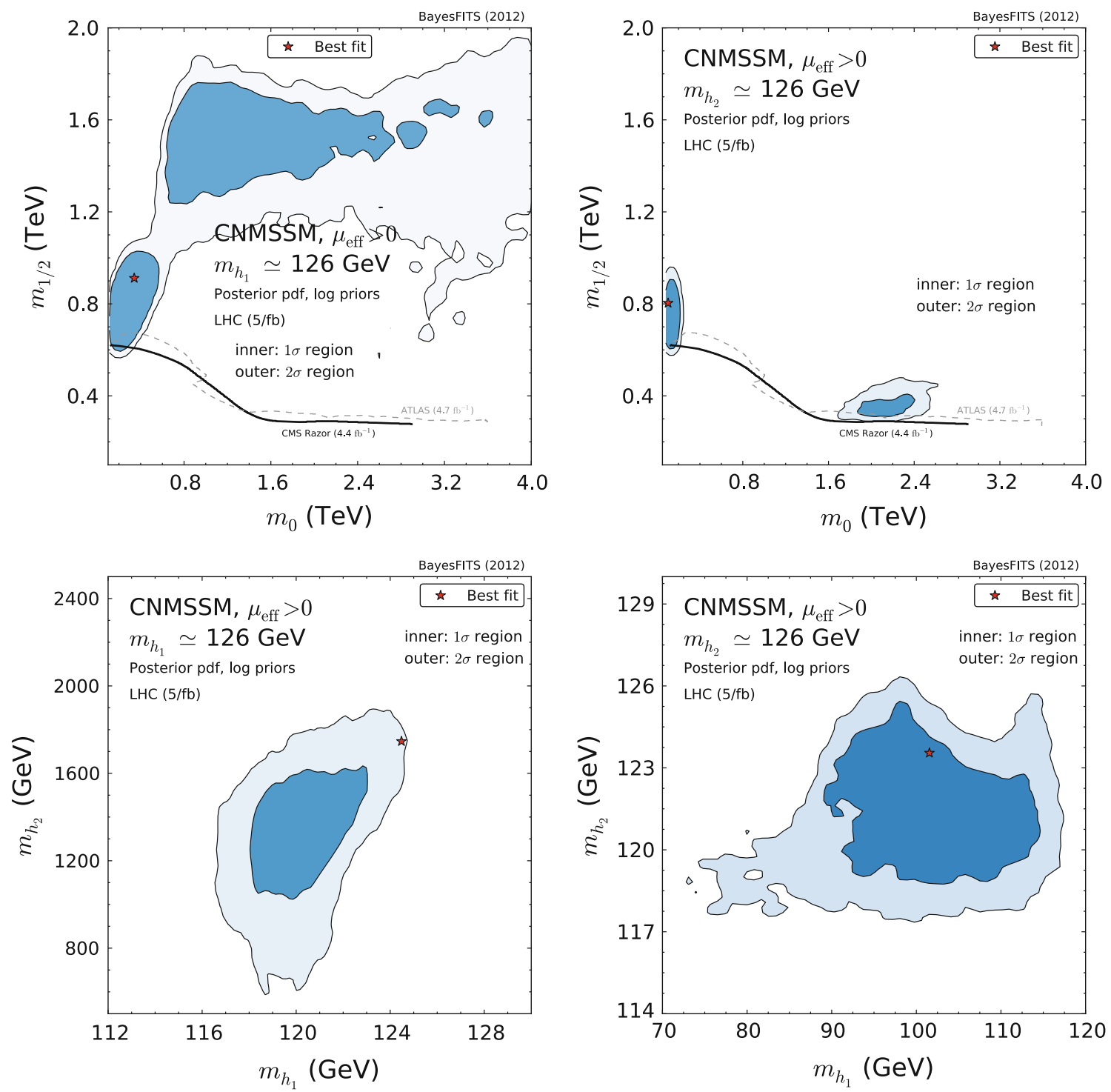

Fig. 2 CNMSSM marginalised $2 \mathrm{D}$ posterior pdf in the $\left(m_{0}, m_{1 / 2}\right)$ plane for case 1 (top left), and case 2 (top right) and in the $\left(m_{h_{1}}, m_{h_{2}}\right)$ plane for case 1 (bottom left), and case 2 (bottom right). The $68 \%$ credible regions are shown in dark blue, and the $95 \%$ credible regions in

light blue. The stars denote the best fit points. In the top figures the solid black (dashed grey) line shows the CMS and ATLAS hadronic $95 \%$ C.L. exclusion bound

The favoured values for the SUSY mass spectrum following from the requirement that the SUSY LSP should provide the observed dark matter abundance gives $m_{\tilde{g}}=$ $2.8 \mathrm{TeV}, \quad m_{\tilde{t}}=2.2 \mathrm{TeV}, \quad m_{\tilde{u}_{L}}=3.0 \mathrm{TeV}$ and $m_{\chi}=$ $600 \mathrm{GeV}$ as the most probable values, slightly lower than that found for the CMSSM, but still in the range that may be beyond LHC14 discovery.

\section{The hierarchy problem}

As these simple models demonstrate the non-observation of SUSY states, or indeed any significant deviations from

the SM, drives the SUSY partner mass scale into the $\mathrm{TeV}$ region. Indeed, if one ignores the hierarchy problem, it can be made arbitrarily high if there is a non-SUSY explanation for dark matter. This emphasises the importance of the hierarchy problem for it is really only the SUSY solution to the hierarchy problem that has led us to expect low-scale SUSY, accessible to LHC discovery.

A very simple illustration of the hierarchy problem was given in [119] and follows from expanding the formula for the $Z$ mass in Eq. (3) in terms of the input parameters. Allowing for different gaugino and scalar masses at the input scale, $M_{X}$, and simplifying by working at tree level one finds, for example for $\tan \beta=2.5$, 


$$
\begin{aligned}
\frac{M_{Z}^{2}}{2}= & -0.87 \mu^{2}\left(M_{X}\right)+3.6 M_{3}^{2}\left(M_{X}\right) \\
& -0.12 M_{2}^{2}\left(M_{X}\right)+0.007 M_{1}^{2}\left(M_{X}\right) \\
& -0.71 m_{H_{U}}^{2}\left(M_{X}\right)+0.19 m_{H_{D}}^{2}\left(M_{X}\right) \\
& +0.48\left(m_{Q}^{2}\left(M_{X}\right)+m_{U}^{2}\left(M_{X}\right)\right) \\
& -0.34 A_{t}\left(M_{X}\right) M_{3}\left(M_{X}\right)-0.07 A_{t}\left(M_{X}\right) M_{2}\left(M_{X}\right) \\
& -0.01 A_{t}\left(M_{X}\right) M_{1}\left(M_{X}\right)+0.09 A_{t}^{2}(0) \\
& +0.25 M_{2}\left(M_{X}\right) M_{3}\left(M_{X}\right)+0.03 M_{1}\left(M_{X}\right) M_{3}\left(M_{X}\right) \\
& +0.007 M_{1}\left(M_{X}\right) M_{2}\left(M_{X}\right) .
\end{aligned}
$$

One may see that for the input parameters much greater than $M_{Z}$ it is necessary to fine tune the parameters to arrange for significant cancellation between the terms. For the case these parameters are at the $\mathrm{TeV}$ scale this fine tuning is more than 1 part in 100. This is the hierarchy problem and it clearly gets worse as the input parameters increase.

Of course if the underlying theory relevant at $M_{X}$ requires correlations between the input parameters the fine tuning may be reduced. As an example of this we consider the CMSSM case with universal scalar masses $m_{0}$ and universal gaugino masses $m_{1 / 2}$. In this case Eq. (8) becomes

$$
\begin{aligned}
\frac{M_{Z}^{2}}{2}= & -0.87 \mu^{2}\left(M_{X}\right)+3.78 m_{1 / 2}^{2}+0.44 m_{0}^{2} \\
& -0.42 A_{0} m_{1 / 2}+0.09 A_{0}^{2} .
\end{aligned}
$$

It is instructive to consider why the coefficient of $m_{0}$ is relatively small in the CMSSM. The dominant terms in the RG equation for $m_{H_{u}}^{2}$, which sets the EW scale, involving $m_{0}$ are those proportional to the square of the top Yukawa coupling, $y_{t}$ and can be integrated to give

$$
\begin{aligned}
& m_{H_{u}}^{2}\left(Q^{2}\right)=m_{H_{u}}^{2}\left(M_{X}^{2}\right)+\frac{1}{2}\left(m_{H_{u}}^{2}\left(M_{X}^{2}\right)\right. \\
& \left.\quad+m_{Q_{3}}^{2}\left(M_{X}^{2}\right)+m_{\bar{u}_{3}}^{2}\left(M_{X}^{2}\right)\right)\left[\left(\frac{Q^{2}}{M_{X}^{2}}\right)^{\frac{3 y_{t}^{2}}{4 \pi^{2}}}-1\right] \\
& =m_{0}^{2}\left(1+\frac{3}{2}\left[\left(\frac{Q^{2}}{M_{X}^{2}}\right)^{\frac{3 y_{t}^{2}}{4 \pi^{2}}}-1\right]\right),
\end{aligned}
$$

where we have used the fact that in the CMSSM all the scalar masses are equal unification scale. When the factor in square brackets is $-2 / 3$ the coefficient of $m_{0}^{2}$ vanishes-this is known as the focus point (FP) [120-124]. Remarkable the focus point is close to the electroweak scale explaining the smallness of the coefficient of $m_{0}^{2}$ in Eq. (9). Clearly the appearance of such focus points affect the bounds on the SUSY spectrum coming from the hierarchy problem because the dependence of the other scalars, the squarks and sleptons, on $m_{0}^{2}$ is not suppressed and consequently, for models with the focus point, they can be much heavier than the Higgs.
This nicely illustrates how correlations amongst the initial parameters can significantly reduce the fine tuning needed.

\subsection{Fine tuning measures}

In order to quantify the fine tuning needed to keep the electroweak scale much lower than the SUSY masses several fine tuning measures have been suggested $[125,126]$. Two frequently used are $\Delta_{m}$ and $\Delta_{q}$ where

$$
\begin{aligned}
& \Delta_{m}=\max \left|\Delta_{\gamma_{i}}\right|, \quad \Delta_{q}=\left(\sum \Delta_{\gamma_{i}}^{2}\right)^{1 / 2}, \\
& \Delta_{\gamma_{i}}=\frac{\partial \ln v^{2}}{\partial \ln \gamma_{i}^{2}}, \quad \gamma_{i}=m_{0}, m_{1 / 2}, \mu_{0}, A_{0}, \ldots
\end{aligned}
$$

Here the basic measure $\Delta_{\gamma_{i}}$ roughly determines the relative magnitude of the term proportional to the parameter $\gamma_{i}$ on the RHS of Eq. (8) to the LHS. A value of 100 means that the cancellation between terms on the RHS should be accurate to 1 part in 100 . Typically one term dominates in which case $\Delta_{m}$ and $\Delta_{q}$ are nearly equal but in the case that there are several comparable terms $\Delta_{q}$ would seem the more reasonable measure.

\subsection{The likelihood origin of the fine tuning measure}

Of course the difficult question to answer when using such measures to limit the SUSY spectrum is how large the fine tuning measure can reasonably be? However, it has recently been shown how the measure arises when performing a likelihood fit to the data [127-130] and this allows us to give a quantitative estimate for acceptable fine tuning.

When testing a SUSY model with a given set of parameters (such as $\gamma_{i}$ ), one should in principle marginalise (i.e. integrate) the likelihood $L$ over unrelated, 'nuisance' parameters that can be determined well from the data and are not of interest in the final result. Examples of such parameters are those already present in the Standard Model such as the Yukawa couplings. In the case of SUSY one may also integrate over the dependent parameters, the VEVs $v_{u, d}$ of the SUSY Higgs $H_{u, d}$ which are fixed by the vacuum minimisation constraints and which determine the EW breaking scale characterised by $M_{Z}$. If one chooses to do this, then the constrained likelihood is given by

$$
\begin{aligned}
L\left(\text { data } \mid \gamma_{i}\right) \propto & \int \mathrm{d} v \mathrm{~d}(\tan \beta) \delta\left(m_{Z}-m_{Z}^{0}\right) \\
\times & \delta\left(f_{1}\left(\gamma_{i} ; v, \beta, y_{t}, y_{b}\right)\right) \delta\left(f_{2}\left(\gamma_{i} ; v, \beta, y_{t}, y_{b}\right)\right) \\
& L\left(\text { data } \mid \gamma_{i} ; v, \beta, y_{t}, y_{b}\right) .
\end{aligned}
$$

where $v^{2}=v_{u}^{2}+v_{d}^{2}$ and $\tan \beta=v_{u} / v_{d} . L\left(\right.$ data $\mid \gamma_{i} ; \beta, v$, $\left.y_{t}, y_{b}\right)$ is the likelihood to fit the data with a particular set of values for $\gamma_{i}, y_{t, b}$, etc. (the associated $\chi^{2}$ is given by $\chi^{2}=$ $-2 \ln (L)$ ), while $L$ (data| $\gamma_{i}$ ) is the ('constrained') likelihood 
in the presence of the EW constraints and is a function of $\gamma_{i}$ only. The functions $f_{i}$ are the solutions to the minimisation equations given by

$$
\begin{aligned}
f_{1}\left(\gamma_{i} ; v, \beta, y_{t}, y_{b}, \ldots\right) \equiv & v-\left(-\frac{m^{2}}{\lambda}\right)^{1 / 2}, \\
f_{2}\left(\gamma_{i} ; v, \beta, y_{t}, y_{b}, \ldots\right) \equiv & \tan \beta-\tan \beta_{0}\left(\gamma_{i}, v, y_{t}, y_{b}\right), \\
& \gamma_{i}=\left\{m_{0}, m_{1 / 2}, \mu_{0}, A_{0}, B_{0}\right\},
\end{aligned}
$$

where $m^{2}$ and $\lambda$ are the effective Higgs mass and quartic coupling, functions of the underlying parameters of the theory as in [127].

Of particular relevance here is the delta function involving $f_{1}$ which constrains some combination of the parameters of the theory. We may choose the residual independent variables to lie in the surface, $S$, in $\gamma_{\kappa}$ space defined by the EW constraint $f_{1}\left(\gamma_{i}^{S} ; v_{0}, \beta, \tilde{y}_{t}(\beta), \tilde{y}_{b}(\beta)\right)=0$. In this case we have $\delta\left(f_{1}\left(z_{i}\right)\right)=\left(1 /\left|\nabla f_{1}\right|\right) \delta\left[\vec{n} \cdot\left(\vec{z}-\vec{z}^{S}\right)\right]$, where $\vec{n}=\nabla f_{1} /\left|\nabla f_{1}\right|$ is the unit vector normal to the surface $S$, $\vec{z}$ has components $z_{1}, \ldots, z_{n}$ and $\vec{z}^{S}$ lies on $S, f\left(z_{i}^{S}\right)=0$. With this, and taking $z_{i}=\ln \gamma_{i}$, Eq. (13) can be written as

$$
\begin{aligned}
L\left(\text { data } \mid \gamma_{i}\right)= & \frac{1}{\Delta_{q}} \delta\left(\sum_{i=1}^{n} n_{q}\left(\ln \gamma_{i}-\ln \gamma_{i}^{S}\right)\right) \\
& \times\left. L\left(\operatorname{data} \mid \gamma_{i} ; v_{0}, \beta, \tilde{y}_{t}(\beta), \tilde{y}_{b}(\beta)\right)\right|_{\beta=\beta_{0}\left(\gamma_{i}\right)}
\end{aligned}
$$

where $n_{q}$ are the components of the normal unit vector and $\Delta_{q}$ is the fine tuning in quadrature defined in Eq. (12) with $v \rightarrow \tilde{v}$. For independent variables, $\gamma_{i}$, this relation is only satisfied if all $\gamma_{i}=\gamma_{i}^{0}$, for all $i$, i.e. if the $f_{1}=0$ constraint is satisfied.

This shows that it is the constrained likelihood that should be maximised when fitting data, i.e. one should maximise the ratio of the unconstrained likelihood to the fine tuning $\Delta_{q}$. If the fine tuning is large it reduces the overall likelihood. The terms of the associated $\chi^{2}\left(\chi_{\text {new }}^{2}\right)$ and unconstrained $\left(\chi_{\text {old }}^{2}\right)$ likelihoods are related by

$\chi_{\text {new }}^{2}=\chi_{\text {old }}^{2}+2 \ln \Delta$.

This relation can be used to infer what can be regarded as the 'acceptable' upper bound of the fine tuning requiring that $\Delta_{q} \ll \exp \left(n_{d} / 2\right)$ where $n_{d f}$ is the number of degrees of freedom. If this is satisfied then $\chi^{2}$ per degree of freedom will not be significantly worsened. For simple SUSY extensions of the SM such as the CMSSM $n_{d f}=O(10)$, which requires $\Delta \ll 100^{5}$.

\footnotetext{
$\overline{5 \text { See }[128,129}]$ for a detailed analysis of various SUSY models.
}

3.3 The real hierarchy problem

We have introduced the hierarchy problem in the context of supersymmetry but, given the lack of evidence for BSM physics at the LHC or in precision tests of the SM, it may be appropriate to make a few comments about claims that, even in the context of the SM, there is no hierarchy problem. It has become customary to introduce the hierarchy problem by noting that in the SM at leading order there is a quadratically divergent contribution to the Higgs mass coming from a top quark loop giving

$\delta m_{H_{u}}^{2} \sim-\frac{3\left|y_{t}^{2}\right|}{4 \pi^{2}} \Lambda^{2}$.

In a renormalisable theory this divergent term is cancelled by a counter term but, taking the cutoff to be at a very high scale, this cancellation must be hierarchically precise. However, the quadratic divergence is not the real hierarchy problem in the sense that the divergent term is independent of the scale at which the mass is measured. Thus if, at a very high scale, there is a reason, such as underlying scale invariance or SUSY, for there to be no mass term it will not reappear at lower scales. The real hierarchy problem [131] arises when there are heavy states of mass $M_{X}$ to which the Higgs couples which give corrections of the form

$\delta m_{H_{u}}^{2}\left(Q^{2}\right) \propto y^{2} M_{X}^{2} \ln \left(\frac{\Lambda^{2}}{M_{X}^{2}+Q^{2}}\right)$.

Even if the mass vanishes at a high scale it reappears at a lower scale and, for it to be small at the electroweak scale, requires a precise cancellation with other terms. In a supersymmetric model this cancellation is between contributions involving different components of a supermultiplet and is automatic due to the symmetry. Thus in a SUSY GUT the term in Eq. (18) is cancelled up to terms suppressed by $\Delta_{\text {SUSY }}^{2} / M_{X}^{2}$ where $\Delta_{\text {SUSY }}$ is the SUSY breaking scale in the GUT sector. For the case of scale invariance the idea is that it is softly broken so that at high energy scales the breaking terms are small and the underlying scale invariance ensures that the quadratic terms is cancelled by the counter term. However, as the SM is not scale invariant its beta functions do not vanish and thus must change at some scale, $\mathrm{M}$, to the scale invariant result. Even if the origin of the mass scale, M, is non-perturbative it has been shown recently [132] that the Higgs mass has corrections of $O(M)$. Thus to protect the Higgs mass, the scale $M$, corresponding to the onset of BSM physics, is limited to the $\mathrm{TeV}$ scale, otherwise the hierarchy problem reappears.

\subsection{Fine tuning and the initial scale}

For pedagogic reasons we introduced the fine tuning measure in the context of the CMSSM in which the low energy 
structure of the MSSM spectrum is determined by parameters defined at a high (unification) scale. Such high scale models usually introduce large logarithmic enhancements in the fine tuning measure coming from the RG continuation to the electroweak breaking scale. As is exemplified by the scalar focus point structure discussed above the logarithmic enhancement may be significantly reduced if there are relations between the SUSY breaking parameters that an underlying theory might provide. It is perhaps interesting to ask what is a reasonable estimate for the minimum fine tuning that could come from a (presently unknown) underlying theory providing such relations. To quantify this, note that in the MSSM the electroweak scale, characterised by the $Z$ mass, is given by

$\frac{M_{Z}^{2}}{2}=\frac{m_{H_{d}}^{2}+\Sigma_{d}^{d}-\left(m_{H_{u}}^{2}+\Sigma_{u}^{u}\right) \tan ^{2} \beta}{\tan ^{2} \beta-1}-\mu^{2}$

where $\Sigma_{u}^{u}$ and $\Sigma_{d}^{d}$ are one-loop contributions coming from the states that couple to the Higgs. Requiring that the theory be parameterised by just these terms, with no information about the underlying UV completion, leads to the 'EW' fine tuning measure [133-136] given by the maximum value of the individual terms ${ }^{6}$. We have

$$
\begin{aligned}
& \Delta_{\mathrm{EW}}=\operatorname{Max} \\
& \quad \times\left(\left|\frac{\mid m_{H_{u}}^{2} \tan ^{2} \beta}{\tan ^{2} \beta-1}\right|,\left|\frac{\Sigma_{u}^{u} \tan ^{2} \beta}{\tan ^{2} \beta-1}\right|, \ldots,\left|\mu^{2}\right|\right) /\left(M_{Z}^{2} / 2\right) .
\end{aligned}
$$

In this case one can take squark and gluino masses to be very heavy because only $\Sigma_{u}^{u}$ and $\Sigma_{d}^{d}$ are sensitive to the radiative corrections. The dominant correction comes from the top squark loop

$\Sigma_{u}^{u} \approx \frac{3 y_{t}^{2}}{16 \pi^{2}} m_{\tilde{t}}^{2}\left(\ln \left(m_{\tilde{t}}^{2} / Q^{2}\right)-1\right)$.

The EW fine tuning is determined by the SUSY spectrum and, for acceptable fine tuning, $\Delta_{\mathrm{EW}}<30$, one can have stop (and other) squarks with mass as high as $5 \mathrm{TeV}$. Further it requires $|\mu|<300 \mathrm{GeV}$ corresponding to the existence of light Higgsino states that may be visible at an $e^{+} e^{-}$collider with $E_{\mathrm{CM}}>600 \mathrm{GeV}$ producing $\tilde{W}_{1}^{+} \tilde{W}_{1}^{-}, \tilde{Z}_{1} \tilde{Z}_{1}$ and $\tilde{Z}_{2} \tilde{Z}_{2}$ production. Gluinos can also be very heavy in which case the small mass gap of $O(10 \mathrm{GeV})$ between $\tilde{W}_{1}-\tilde{Z}_{2}$ and $\tilde{Z}_{2}-\tilde{Z}_{1}$ makes detection of Higgsino pair production difficult because the visible decay products are soft.

A low scale of EW fine tuning does not mean there is no fine tuning but leaves open the possibility that there is an underlying theory with this SUSY spectrum that is not fine

\footnotetext{
${ }^{6}$ Of course, this assumes that the underlying theory does not correlate the terms in this equation. To date no theory capable of doing this has been proposed.
}

tuned. Below we discuss UV complete models that do predict correlations among the MSSM SUSY breaking parameters which can achieve such low levels of fine tuning while preserving the successful prediction following from gauge coupling unification. In these models the mass of the coloured states are much more constrained than the upper bound coming from EW fine tuning. They do, however, have the tendency to produce compressed spectra.

\section{Fine tuning constraints on SUSY in the light of the Higgs discovery}

As we have stressed the hierarchy problem provides the main motivation for low-scale SUSY, accessible to the LHC. In this section we will discuss the extent to which the postulate that SUSY solves the little hierarchy problem has been tested ${ }^{7}$ and the prospects for a definitive test at LHC14. This will involve a discussion of non-minimal implementations of SUSY that can reduce the fine tuning and, in turn, suggest new signatures relevant to future LHC SUSY searches. In the main we will concentrate on the case that the initial scale at which the fundamental parameters are defined is close to the Grand Unified scale as is natural in GUTs and is consistent with the precision prediction of gauge coupling unification that follows in SUSY GUTs.

\subsection{Fine tuning of the CMSSM}

In the CMSSM the Higgs mass is given by Eq. (5). The heavier the Higgs mass is the larger the radiative correction that is needed. Before the LHC start-up the bound on the Higgs mass was $114 \mathrm{GeV}$ corresponding, for small $X_{t}$, to $M_{S} \approx 500 \mathrm{GeV}$. The measurement of the Higgs mass close to $126 \mathrm{GeV}$ increases this to $M_{S} \approx 1 \mathrm{TeV}$. Thus the Higgs discovery has pushed the SUSY threshold for the stops up and this leads to the need for significantly greater fine tuning. Of course one must also allow for the $X_{t}$ contribution and perform a fit to all the available data. The result of such a fit [138] that was performed before the LHC start-up is shown in Fig. 3 where the fine tuning, $\Delta \equiv \Delta_{m}$, is shown as a function of the Higgs mass; note that the LEP bound on the Higgs mass was not included in the fit. The origin of the structure is due to two factors: the fall as the Higgs mass increases is due to the fact that the effective quartic interaction, $\lambda_{\text {eff }}$, increases, reducing the sensitivity of the EW breaking VEV, $v^{2}=m_{\text {eff }}^{2} / \lambda$ eff to changes in $\lambda_{\text {eff }}$. The sharp rise as the Higgs mass further increases is due to the fact that the sensitivity of $m_{H_{u}}$ to $m_{0}$ in Eq. (11) increases rapidly as $Q^{2} \sim m_{H_{u}}^{2}$ grows above $(115 \mathrm{GeV})^{2}$. Also shown in the figure is the

\footnotetext{
${ }^{7}$ For an earlier comparative study see [137].
} 


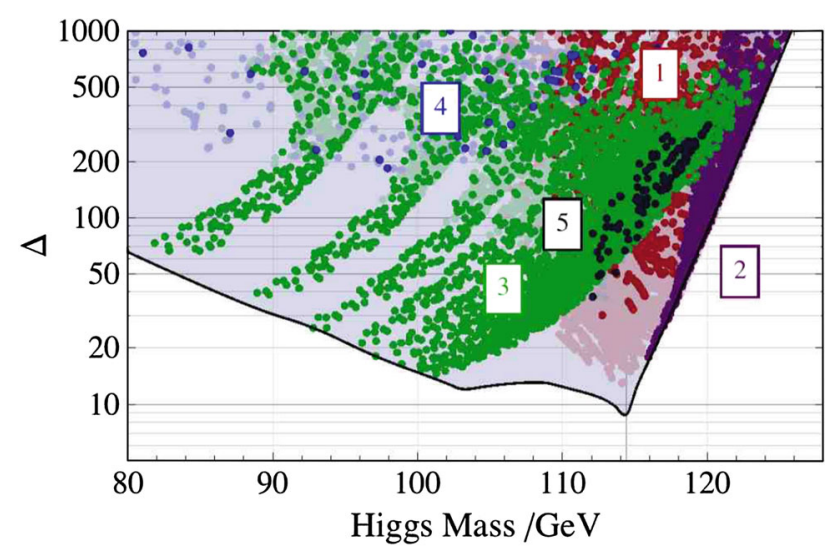

Fig. 3 Two-loop fine-tuning versus Higgs mass for the scan over CMSSM parameters with no constraint on the Higgs mass. The solid line is the minimum fine-tuning with $\left(\alpha_{s}, M_{t}\right)=(0.1176,173.1 \mathrm{GeV})$. The dark green, purple, crimson and black coloured regions have a dark matter density within $\Omega h^{2}=0.1099 \pm 3 \times 0.0062$ (i.e. $3 \sigma$ saturation) while the lighter coloured versions of these regions lie below this bound. The colours and their associated numbers refer to different LSP structures as described in [138]. Regions 1, 3, 4 and 5 have an LSP which is mostly bino-like. In region 2, the LSP has a significant Higgsino component

dark matter abundance colour coded [138] according to the dominant annihilation mechanism. The purple points with low fine tuning lie close to the focus point discussed above and one may see that before the LHC there are points in the parameter space scan with fine tuning less than 10 , close to the LEP bound on the Higgs mass. As noted above, the points in the FP region have significant Higgsino component and the lowest fine tuned points are in conflict with the XENON100 bounds. However, the most significant effect ruling out the low fine tuned points is the measurement of the Higgs mass. For a Higgs mass in the range $m_{h}=126 \pm 3 \mathrm{GeV}$ the fine tuning, $\Delta_{m}$, is greater than 300 , unacceptably large given the constraint of Eq. (16).

\subsection{Beyond the CMSSM}

Of course the CMSSM is only one particular version of the MSSM expressing the more than 100 SUSY parameters in terms of just 5 fundamental ones. One may ask if there are other MSSM parameter choices with lower fine tuning that remain to be tested. However, this is not so easy as the CMSSM has the scalar focus point that, cf. Eq. (11), desensitises the EW breaking scale to the common scalar mass $m_{0}$ and in this sense represents the class of models capable of minimising, at least part, of the fine tuning measure. In contrast, gauge mediated supersymmetry-breaking models do not have a common scalar mass and as a result the fine tuning in them may be larger $[139,140]$ even though they may have a lower initial scale, $M_{X}$.

To do better than the CMSSM requires identifying a systematic way to reduce fine tuning. In this subsection we discuss whether the fine tuning can be reduced by theoretically well-motivated modifications of the CMSSM boundary conditions for the SUSY breaking parameters. In the next subsection we consider the possibility that the fine tuning is reduced through an extension of the particle content of the MSSM.

\subsubsection{Natural SUSY}

In natural SUSY the universality of squark masses is relaxed with much lighter stop squarks than those associated with the first two generations $[141,142]$. As we discuss in Sect. 5 the suppression of flavour-changing neutral currents and CPviolating effects place strong constraints on the first and second generation squarks favouring their mass to be in the $\mathrm{TeV}$ region. However, the constraint on the stop squarks is very mild and this has led to the suggestion that they may be quite light, much less than a TeV. This is consistent with present LHC bounds due to the reduction in the $E_{T}$ missing signals compared to that for the first two generation squarks. Since a large contribution to fine tuning comes from the sensitivity of the EW scale to the stop quark mass one may hope that fine tuning will be substantially reduced. However, this turns out not to be the case because it is still necessary to have significant radiative corrections to the Higgs mass to drive it to $126 \mathrm{GeV}$ and, for light stops, this must come from another sector of the theory, reintroducing large fine tuning. Recent studies [143,144] finds the fine tuning is at least 400 for the case the initial scale, $M_{X}$, at which the parameters are defined is close to the GUT scale, unacceptably large by the criterion in Eq. (16). Even in the case that the initial scale is low, such as in gauge mediation, there is no significant fine tuning advantage of a light stop if the gluino is in the $\mathrm{TeV}$ range and the fine tuning is still in the $1 \%$ range. Interestingly [143] notes that this conclusion can be evaded in the case that the gluinos have a Dirac rather than a Majorana mass although a very recent study [144] has found the fine tuning is still severe, $\Delta \sim 100$.

\subsubsection{Gaugino focus point}

The second possibility that has been suggested is that there is a further focus point associated with the gauginos that reduces the sensitivity of the EW breaking scale to $m_{1 / 2}{ }^{8}$. This can occur if the initial values of the gaugino masses have special, non-universal, ratios [145-155]. The origin of the gaugino focus point may be seen from the RG equation

\footnotetext{
8 As we shall discuss this can reduce the fine-tuning to an acceptable level. This conflicts with the conclusion of [144] which does not allow for such correlations between soft SUSY breaking terms.
} 

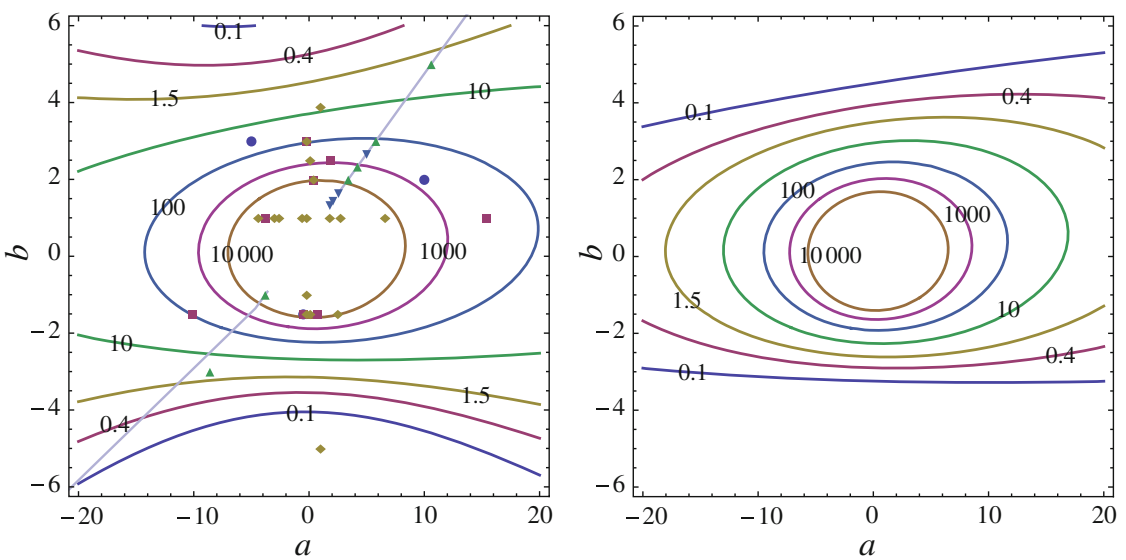

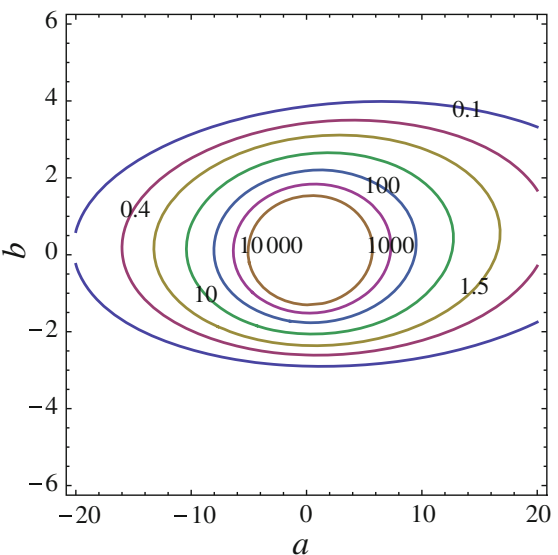

Fig. 4 Analytic results for the gaugino focus point scale contours (in units of Tev) in the MSSM for $\tan \beta=2,3,10$ from left to right. The points and the grey line correspond to specific models with non- universal gaugino masses; see text for details. The contours do not change much for larger $\tan \beta$

tations of 'flipped $\mathrm{SO}(10)$ ' embedded in $E_{6}$ predict gaugino mass ratios in the intermediate and low fine tuning region. Green triangles represent the OII orbifold model for various choices of the discrete Green Schwarz parameter, $\delta_{\mathrm{GS}}$ [158]. The values $\delta_{\mathrm{GS}}=-5,-6,-7$ are optimal from the point of view of fine tuning. For comparison we also show points relevant for mirage mediation $[147,159,160]$, where soft terms receive comparable contributions from gravity (modulus) and anomaly mediated SUSY breaking. In this case gaugino masses at the GUT scale have the following form:

$M_{a}=m_{3 / 2}\left(\varrho+b_{a} g_{a}^{2}\right)$

where $g_{a}$ is the relevant gauge coupling, $b_{a}$ is its $\beta$-function coefficient, while $\varrho$ describes the relation between modulus and anomaly mediated contributions. This prescription for gaugino masses as a function of $\varrho$ generates the grey line in Fig. 4 in the $(a, b)$ parameter space. If $\varrho$ is a continuous parameter there should be an additional contribution $\Delta_{\varrho}$ to the overall fine tuning. However, specific string models fix the value of $\varrho$. Four examples are shown in Fig. 4 by the blue inverted triangles:

(i) the minimal setup of KKLT-type moduli stabilisation in type II B string theory [161-163],

(ii) a model with vacuum uplifting via hidden sector matter superpotentials [161],

(iii) and (iv) the Mini Landscape of orbifold compactifications in heterotic string theory [164] with SU(4) and SU(5) hidden sector gauge groups; the type II B string theory model with vacuum stabilisation by $\mathrm{F}$ terms of hidden sector matter superpotentials predicts values of $a$ and $b$ in the low fine tuning region.

Given that there are models that naturally have the gaugino focus point at the EW scale it is interesting to ask how the 


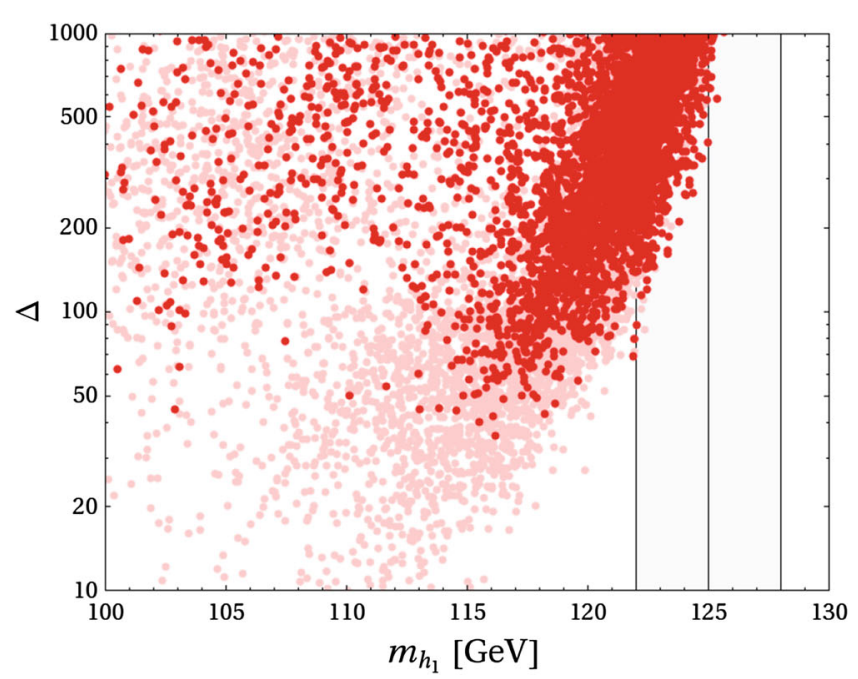

Fig. 5 Dependence of the fine tuning on the Higgs mass. The light red points are before any cuts while the dark red points take into account cuts on the SUSY masses and the relic neutralino abundance. The left

overall fine tuning is affected. We consider the case of the MSSM with the CMSSM spectrum modified to allow for non-universal gaugino masses at the unification scale (the (C)MSSM) and requiring gauge coupling unification. The results of a fit to all the available data including the DM abundance is shown in Fig. 5 where it can be seen that there are some points, with $a$ and $b$ in the low fine tuned regions of Fig. 4, with fine tuning less than 100, marginally acceptable by the criterion of Eq. (16).

Due to the additional flexibility in the gaugino sector, a large variety of LSP compositions is possible. For points satisfying the relic abundance upper bound the LSP is mainly composed of wino and Higgsino, with typically only a very small bino component. Unlike the case for the CMSSM the direct detection cross section lies below the Xenon100 limit with the bulk of the points more than two orders of magnitude below. The correct relic abundance seems to be more easily achieved with a Higgsino-like LSP. A recent discussion of the phenomenology of the low fine tuned points can be found in [165].

\subsection{Beyond the MSSM}

The MSSM is the minimal extension of the SM, minimal in the sense that the fewest new states have been included when building a SUSY model. Could it be that non-minimal extensions reduce the fine-tuning constraints on SUSY and have not yet been experimentally tested?

\subsection{Operator analysis}

A useful way to look such extensions is to allow for a general modification of the MSSM by adding higher dimension oper-

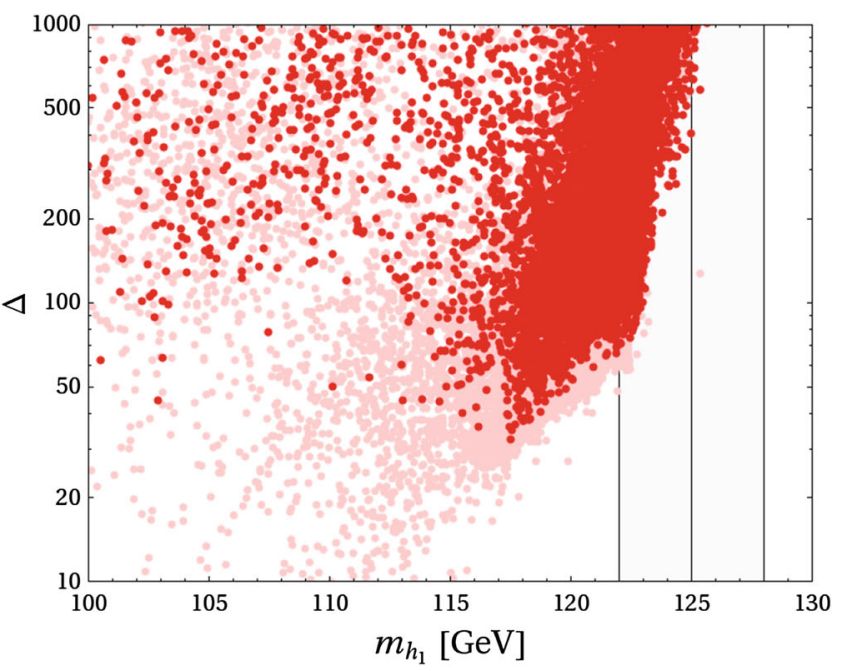

plot is uniform in the density of the input parameters, their density reflects the likelihood for finding a viable point. The right plot shows additional points where we zoomed into regions of small fine tuning

ators [166-173] that correspond to the effective field theory that results from integrating out additional heavy degrees of freedom, of mass $M_{*}$, and ask if such operators can reduce fine tuning. There is a unique leading dimension 5 operator with the form

$L=\frac{1}{M_{*}} \int \mathrm{d}^{2} \theta f(X)\left(H_{u} H_{d}\right)$

where $X=\theta \theta m_{0}$ and $m_{0}$ is the SUSY breaking scale.

This gives contributions to the scalar potential of the form

$$
\begin{aligned}
V= & \left(\left|h_{u}\right|^{2}+\left|h_{d}\right|^{2}\right)\left(\chi_{1} h_{u} h_{d}+\text { h.c. }\right) \\
& +\frac{1}{2}\left(\chi_{2}\left(h_{u} h_{d}\right)^{2}+\text { h.c. }\right)
\end{aligned}
$$

where $\chi_{1}=2 f(0) \mu_{\mathrm{eff}} / M_{*}, \chi_{2}=-2 f^{\prime}(0) m_{0} / M_{*}$ and $\mu_{\mathrm{eff}}$ is the effective $\mu$ term. Note that the $\chi_{1}$ term is supersymmetric so there are associated corrections involving Higgsinos that will generate Higgsino mass terms of the same order of magnitude as the correction to the Higgs mass terms (once the Higgs acquire their VEVs). However, in practice these corrections are going to be of $O(10 \mathrm{GeV})$, important to get a Higgs mass of $125 \mathrm{GeV}$ but small compared to the Higgsino mass coming from the $\mu_{\text {eff }}$ term. For this reason we concentrate on the effect in the scalar sector.

The fine tuning of this model has been analysed in [174] where it was shown that the fine tuning is significantly reduced by the first term of Eq. (25) while the second term only gives a modest reduction. The dominant effect comes from the contribution of Eq. (25) to the Higgs mass after electroweak breaking and, due to the fact that the first term involves an extra power of $h_{u}$, it gives the larger contribution.

The obvious question is what new physics can give rise to the first operator corresponding to this term. The answer is 
through the integration out of a new heavy gauge singlet or SU(2) triplet superfield coupling to the Higgs sector. Interestingly the operator is not generated in the NMSSM, the simplest singlet extension of the MSSM, as it requires an explicit mass term for the singlet super field ${ }^{10}$ We refer to this model as the generalised NMSSM (the GNMSSM).

\subsection{The GNMSSM superpotential}

The most general extension of the MSSM by a gauge singlet chiral superfield consistent with the SM gauge symmetry has a superpotential of the form

$$
\begin{aligned}
\mathcal{W} & =\mathcal{W}_{\text {Yukawa }}+\frac{1}{3} \kappa S^{3}+(\mu+\lambda S) H_{u} H_{d}+\xi S+\frac{1}{2} \mu_{s} S^{2} \\
& \equiv \mathcal{W}_{\mathrm{NMSSM}}+\mu H_{u} H_{d}+\xi S+\frac{1}{2} \mu_{s} S^{2}
\end{aligned}
$$

where $\mathcal{W}_{\text {Yukawa }}$ is the MSSM superpotential generating the SM Yukawa couplings and $\mathcal{W}_{\text {NMSSM }}$ is the 'normal' NMSSM with a $Z_{3}$ symmetry. One of the dimensionful parameters can be eliminated by a shift in the VEV $v_{s}$. We use this freedom to set the linear term in $S$ in the superpotential to zero, $\xi=0$.

The form of Eq. (12) seems to make the hierarchy problem much worse as the SM symmetries do not prevent arbitrarily high scales for the dimensionful mass terms. However, these terms can be naturally of the order of the SUSY breaking scale if there is an underlying $Z_{4}^{R}$ or $Z_{8}^{R}$ symmetry $[176,177]$. Before SUSY breaking the superpotential is of the NMSSM form. However, after supersymmetry breaking in a hidden sector with gravity mediation soft superpotential terms are generated but with a scale of order the supersymmetry-breaking scale in the visible sector characterised by the gravitino mass, $m_{3 / 2}$. With these the renormalisable terms of the superpotential take the form

$$
\begin{aligned}
& \mathcal{W}_{Z_{4}^{R}} \sim \mathcal{W}_{\mathrm{NMSSM}}+m_{3 / 2}^{2} S+m_{3 / 2} S^{2}+m_{3 / 2} H_{u} H_{d}, \\
& \mathcal{W}_{Z_{8}^{R}} \sim \mathcal{W}_{\mathrm{NMSSM}}+m_{3 / 2}^{2} S
\end{aligned}
$$

where the $\sim$ denotes that the dimensional terms are specified up to $\mathcal{O}(1)$ coefficients. Clearly the $Z_{4}^{R}$ case is equivalent to the GNMSSM. After eliminating the linear term in $S$ the $Z_{8}^{R}$ case gives a constrained version of the GNMSSM with $\mu_{s} / \mu=2 \kappa / \lambda$.

Note that the SUSY breaking also breaks the discrete $R$ symmetry but leaves the subgroup $Z_{2}^{R}$, corresponding to the

\footnotetext{
$\overline{10}$ Very recently it has been pointed out [175] that it is possible to enhance the effect of the second term in Eq. (25) for the case that the singlet field acquires a Dirac rather than a Majorana mass. This also leads to a significant reduction in fine tuning even for a singlet with mass in the multi $\mathrm{TeV}$ range.
}

usual matter parity, unbroken. As a result the lightest supersymmetric particle, the LSP, is stable and a candidate for dark matter.

\subsection{Fine tuning in the GNMSSM}

Several groups have recently analysed the fine-tuning implications of the GNMSSM [178-184]. Requiring that the couplings remain in the perturbative domain up to the Planck scale, the resulting fine tuning has been explored in detail for the simplified case of universal boundary conditions for the SUSY breaking parameters (CGNMSSM) $[178,179]$. Note that this goes beyond the operator analysis as we do not require that the singlet mass need not be large compared to the other parameters of the theory and thus cannot be integrated out. However, even allowing for the additional contribution to the Higgs mass coming from the singlet couplings, the regions of this model corresponding to low fine tuning have essentially been ruled out by a combination of LHC nonobservation of SUSY and dark matter (DM) abundance. In particular the DM abundance has to be reduced below the 'over-closure' limit and this is dominantly through stau coannihilation that is only effective for relatively low $m_{0}$ and $m_{1 / 2}$ and hence sparticle masses in the reach of LHC 8 .

For the case of non-universal gaugino masses (the (C) GNMSSM) the situation changes because the LSP can now have significant Wino/Higgsino components that ensures its efficient annihilation. In Fig. 6 we show the results of a scan [184] over the region of parameter space which allow for a rather large Higgs mass corresponding to large $\lambda$, (which implies smallish $\kappa$ and small $\tan \beta$ ). The minimal fine tuning after the cuts were imposed is below 20, perfectly acceptable by the criterion of Eq. (16), and there are significant areas of low fine tuning remaining to be explored by LHC14.

To infer something about the typical phenomenology of the low fine tuned regions we consider viable points with fine tuning below 100. Similar to the case of the CGNMSSM the viable points have a large supersymmetric singlet mass parameter, leading to heavy singlet states. In detail this constraint comes from the need to achieve acceptable electroweak breaking consistent with the universality of scalar masses at the high scale and is why such boundary conditions are inconsistent with the NMSSM. Allowing for nonuniversal Higgs masses solves this problem for the MSSM and in the case of the GNMSSM it will allow for lighter singlet states.

In the universal scalar mass case considered here, the singlet states are always heavy and the dominant effect is the change to the Higgs mass that reduces the fine tuning as was found in the CGNMSSM with universal gaugino masses. However, as mentioned above, the region of parameter space of the CGNMSSM that solves the little hierarchy problem has essentially been ruled out by a combination of LHC non- 


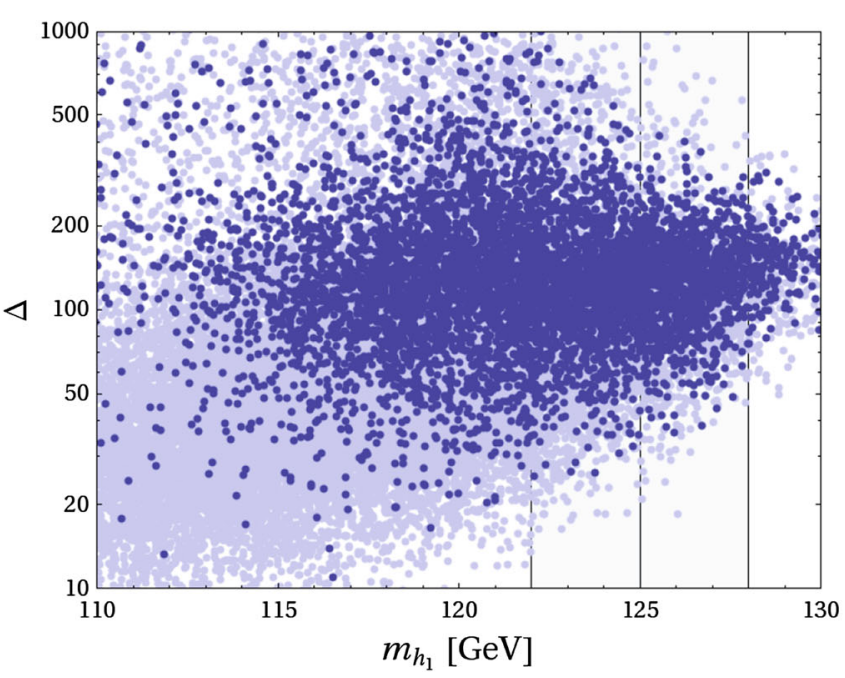

Fig. 6 Dependence of the fine tuning on the lightest Higgs mass in the (C)GNMSSM. The light blue points are before any cuts while the dark blue points take into account cuts on the SUSY masses and the relic neutralino abundance. The left plot is uniform in the density of

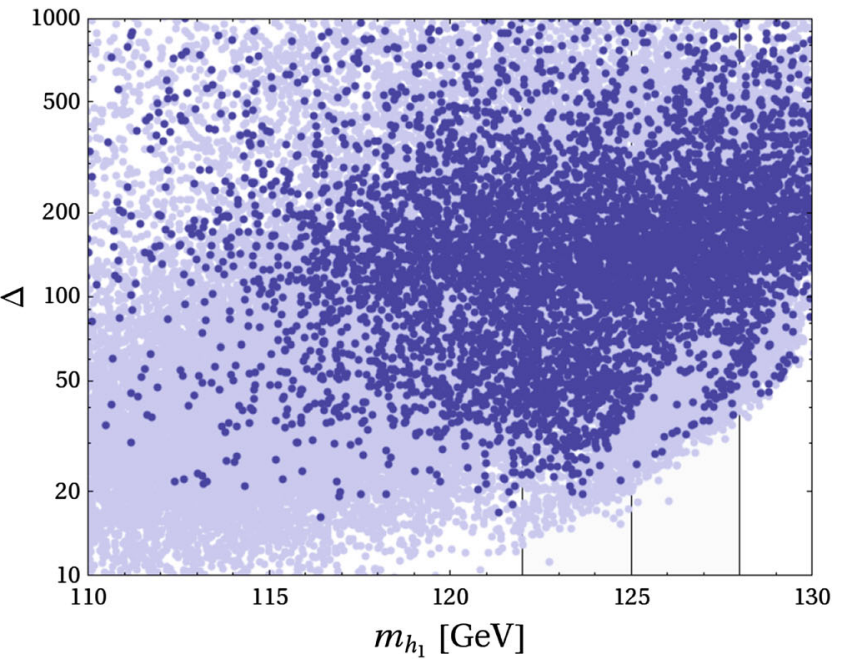

the input parameters, their density reflects the likelihood for finding a viable point. The right plot shows additional points where we zoomed into regions of small fine tuning. The minimal fine tuning after the cuts were imposed is below 20; requiring $a=b=1$ it is about 80

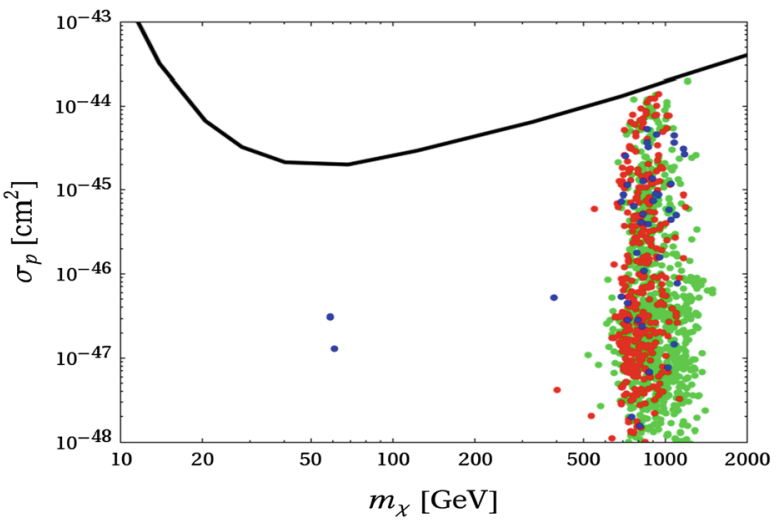

Fig. 7 (i) The dark matter direct detection cross section as a function of the neutralino mass. It has been scaled (i.e. multiplied with $\left(\Omega h^{2}\right)$ th/0.1199) to account for cases with underabundant neutralinos. Also shown is the latest bound from XENON100. (ii) The dark matter composition, that is, the bino, Wino and Higgsino fraction of the LSP, as a function of the relic density. Mostly bino-like LSPs are shown in

observation of SUSY and dark matter abundance. For the case of non-universal gaugino masses the situation changes because the LSP can now have significant non-bino component to allow for its efficient annihilation. In Fig. 7 we show the direct detection cross section versus the mass of the lightest neutralino together with the latest bound from XENON100 as well as the dark matter composition as a function of the relic density. As has been observed in previous studies $[185,186]$ of other models, it may be seen that the mass is in the $\mathrm{TeV}$ range, most often Higgsino-like. It can be seen that almost all of the points are below the XENON100 direct detection limit. Regarding the composition we see that for the correct relic density or an underabundance the LSP is mainly composed of wino and Higgsino, with typically

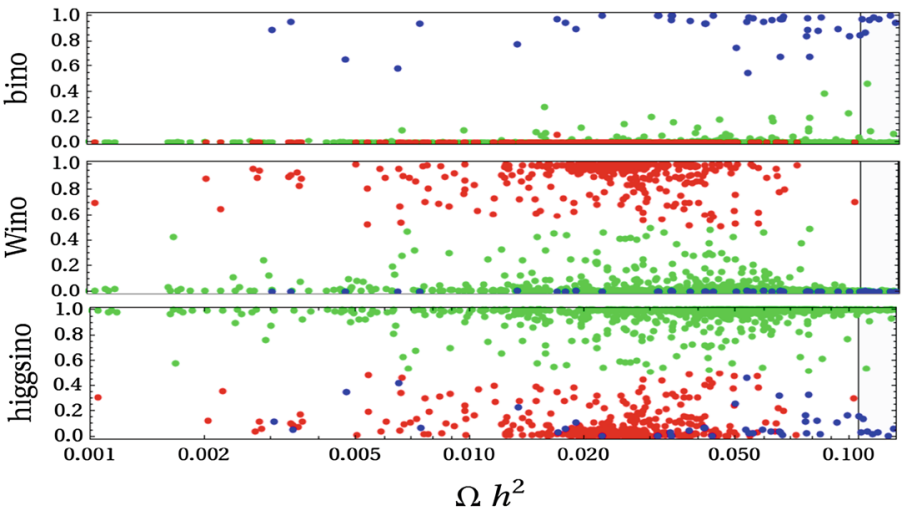

blue, mostly Wino-like LSPs are shown in red and mostly Higgsino-like LSPs are shown in green, where mostly means a fraction $>0.5$. (iii) The distribution of bino-, Wino-, and Higgsino-like LSPs in the $a-b$ plane. For all points, in addition to the SUSY and Higgs cuts, a fine tuning $\Delta<100$ was imposed. The grey points are before the fine tuning cut

only a very small bino component. As in the MSSM the correct relic abundance seems to be more easily achieved with a Higgsino-like LSP. Note that, as is the case for all cases involving universal squark and slepton masses [137], it is not possible to significantly reduce the discrepancy of SM to $g-2$.

In Fig. 8 we show typical masses of the superpartners in the low fine tuned region. It can be seen that points with fine tuning below 100 can have gluino masses beyond $2 \mathrm{TeV}$ and squark masses around $3 \mathrm{TeV}$. The squarks can be much heavier due to a contribution from $m_{0}$. This is limited by the fine tuning implications of a heavy stop unless one is near the scalar focus point. In the CMSSM these points were ruled out by direct dark matter searches as the LSP had signifi- 

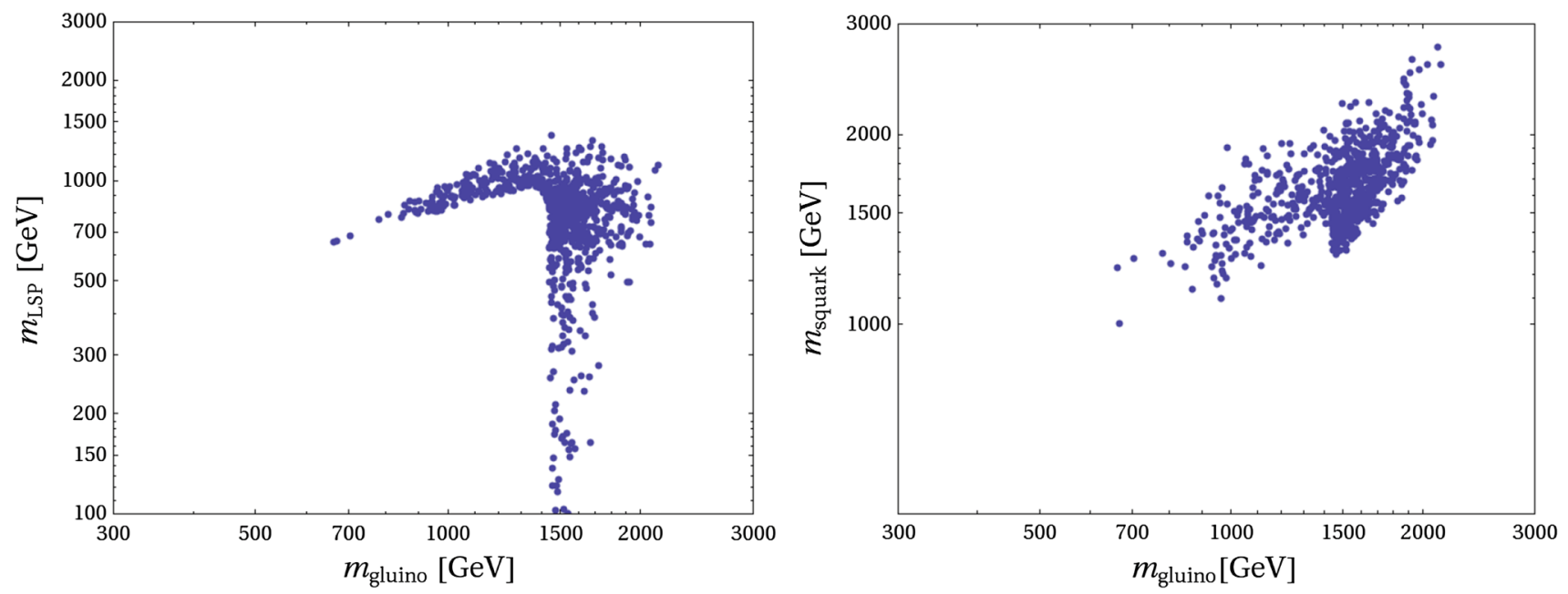

Fig. 8 Mass spectrum of points with fine tuning $\Delta<100$ and all other cuts imposed

cant Higgsino component but in the case of non-universal gauginos this is no longer true. It is also possible that $m_{0}$ is small and the scalar masses are driven by the Gluino and Wino masses. In this case the squark masses will not be much heavier than the gluino although one must worry about sleptons being too light - in particular the neutrino should not be the LSP as this possibility has been ruled out by direct dark matter searches. For the case of heavy squarks the LHC signal is dominantly gluino pair production and decay, but this may be significantly reduced because of the compressed spectrum. Covering the whole of the low fine tuned region will be challenging and will require looking for signals sensitive to compressed spectra [187, 188].

\subsection{Other approaches}

Several other ideas have been suggested to reduce fine tuning. One possibility is that R-parity violation reduces the LHC bounds on SUSY states. This come about because such Rparity violation allows the SUSY state to decay and, if this is dominantly into visible energy, the missing energy signals will be diluted. Of particular interest are the baryon number violating operators $\lambda_{i, j, k} U_{i}^{c} D_{j}^{c} D_{k}^{c}$ that lead to SUSY decay into quarks ${ }^{11}$. Such effects have been widely explored [191203]; however, a recent analysis [144] concludes that the fine tuning is still very large in these models unless, of course, as discussed above one allows for correlations between MSSM parameters and/or an extension of the MSSM spectrum.

Another way of reducing the fine tuning that has been mentioned in Sect. 3.4 is to lower the scale at which the fundamental parameters are defined. This is the case for gauge

\footnotetext{
11 Through simple discrete symmetries $[189,190]$ such terms can arise without the appearance of lepton number violating operators and unacceptable rates of nucleon decay.
}

mediation where the relevant scale is given by the gauge mediator mass. Detailed analyses of realistic models of general gauge mediation $[139,140,204]$ find that the fine tuning may be reduced by a factor of 2 when the mediation scale is reduced from the GUT scale of $O\left(10^{16} \mathrm{GeV}\right)$ to a scale of $O\left(10^{6} \mathrm{GeV}\right)$. This reduction is quite modest and will still require extensions beyond the MSSM of the form discussed above to achieve acceptable fine tuning.

Finally it has been suggested that one should ignore the fine tuning problem altogether [205-207], perhaps appealing to anthropic arguments to justify the existence of a light Higgs capable of generating the EW breaking scale. Even so a case can be made for an underlying SUSY theory with phenomena accessible to the LHC. The reason is that the SUSY gauginos may be lighter than the underlying SUSY breaking scale due to chiral symmetry (an R-symmetry) broken at a lower scale. In this 'split SUSY' case the SUSY scalars, the squarks and the sleptons, are heavy, beyond the LHC reach, while the gauginos are quite light. The gauginos provide the dominant contribution to the difference in the beta functions between the SUSY case and the SM case and thus the success of SUSY gauge coupling unification is maintained ${ }^{12}$. Further, as for conventional SUSY, if the gauginos are light they can provide a viable dark matter candidate. Split SUSY has been widely studied but, due to lack of space and inclination, we do not discuss it further here.

\section{SUSY and flavour physics}

As we have seen the LHC results have significantly increased the lower bound on the masses of SUSY states. This in

\footnotetext{
12 Indeed by adjusting the mass of the heavy SUSY scalars the agreement with the observed gauge couplings can be improved.
} 
turn has implications for flavour physics and it is perhaps timely to re-examine the constraints on SUSY coming from the absence of flavour-changing neutral currents (FCNC) and CP-violating effects [208]. In supersymmetric models flavour-changing and $\mathrm{CP}$-violating effects can be significantly enhanced relative to the SM, driven by processes involving squarks and leptons. In particular such models introduce new sources of $\mathrm{CP}$ violation such as the phase of the $\mu$ term or of the diagonal $A_{0}$ parameters which, if unsuppressed, lead to unacceptable electric dipole moments (EDM) - the SUSY CP problem. They also generate significant contributions to processes such as $K^{0}, \bar{K}^{0}$ mixing and $\mu \rightarrow e \gamma$.

In the latter case the branching ratio for this process is given by (see, e.g., [209])

$$
\begin{aligned}
\mathrm{BR}(\mu \rightarrow e \gamma) \approx & 10^{-6} C\left(\frac{\left|m_{\tilde{\mu}_{R} \tilde{e}_{R}}^{2}\right|}{m_{\tilde{l}_{R}}^{2}}\right)^{2}\left(\frac{100 \mathrm{GeV}}{m_{\tilde{l}_{R}}}\right)^{4} \\
& <2.4 \times 10^{-12},
\end{aligned}
$$

and it is very tightly constrained by the recent measurement [210] as can be seen on the right-hand side. Indeed, for $m_{\tilde{l}_{R}} \sim$ $100 \mathrm{GeV}$ the flavour-changing off-diagonal mass term must be very small, $m_{\tilde{\mu}_{R} \tilde{e}_{R}}^{2} / m_{\tilde{l}_{R}}^{2} \lesssim 10^{-3}$. Clearly if the lepton mass is above $1 \mathrm{TeV}$ this constraint is weakened considerably.

Similarly, the bounds on EDMs impose strong constraints on the SUSY CP-violating phases. In a simplified approach where all relevant phases are taken to be of the same order $\phi$ and all soft masses are set to be of the order $m_{0}$ the different dipole moments are given by $[211,212]$,

$$
\begin{aligned}
d_{e} \sim & 10^{-25} e \mathrm{~cm} \times \sin \phi\left(\frac{300 \mathrm{GeV}}{m_{0}}\right)^{2}\left(\frac{\tan \beta}{3}\right) \\
& <1.05 \times 10^{-27} e \mathrm{~cm} \\
d_{n} \sim & 10^{-24} e \mathrm{~cm} \times \sin \phi\left(\frac{300 \mathrm{GeV}}{m_{0}}\right)^{2}\left(\frac{\tan \beta}{3}\right) \\
< & 2.9 \times 10^{-26} e \mathrm{~cm} \\
d_{H g} \sim & 10^{-26} e \mathrm{~cm} \times \sin \phi\left(\frac{300 \mathrm{GeV}}{m_{0}}\right)^{2}\left(\frac{\tan \beta}{3}\right) \\
& <2.1 \times 10^{-28} e \mathrm{~cm},
\end{aligned}
$$

with the current limits [213-217] given on the right-hand side of the equations, requiring

$$
\frac{1}{\sin \phi} \sim 100\left(\frac{\tan \beta}{3}\right)\left(\frac{300 \mathrm{GeV}}{m_{0}}\right)^{2} \sim 100\left(\frac{300 \mathrm{GeV}}{m_{0}}\right)^{2} .
$$

Again we see that TeV scale SUSY significantly eases the bound on the CP-violating phase although there is still need for small CP-violating phases, $\phi$.
These estimates apply models that have no intrinsic mechanism to solve the flavour problem but the rates are considerably reduced in models in which there is family symmetry to generate viable Yukawa couplings and their related masses and mixing angles. In this case one starts with a CP-invariant theory (compactified 4D theories in string theory often are $\mathrm{CP}$ invariant $-\mathrm{CP}$ being a discrete relic of the higher dimensional Lorentz group). CP is then spontaneously broken by the familon VEVs that spontaneously break the family symmetry. Via the Froggatt Nielsen mechanism the familions generate the CP-violating Yukawa couplings in the flavourchanging sector where it is observed to be large. CP violation in the flavour conserving sector, where it is small, is driven by the flavour changing couplings and consequently is suppressed by powers of small mixing angles. The resulting models do not realise exact minimal flavour violation (MFV) as the soft $A$-terms do not have exactly the same structure as the Yukawa couplings and lead to additional FCNC and $\mathrm{CP}$-violating effects. However, these corrections are also suppressed by powers of small mixing angles.

Detailed estimates for various SUSY models of this type have been made [218-220]. As an example of the expected rates we consider a supergravity model with an SU(3) family symmetry that, while unbroken, guarantees the degeneracy of squarks and sleptons in a given representation of the gauge group ${ }^{13}$. CP-violating and flavour-changing couplings are generated when the symmetry is spontaneously broken. Then the rate for $\mu \rightarrow e \gamma$ characterised by the mass insertion parameter, $\left|\left(\delta_{\mathrm{LR}}^{\ell}\right)_{12}\right|$ [222-224], is given by

$$
\begin{aligned}
\left|\left(\delta_{\mathrm{LR}}^{\ell}\right)_{12}\right| \approx & 1 \times 10^{-4} \frac{A_{0}}{100 \mathrm{GeV}} \frac{(200 \mathrm{GeV})^{2}}{\left\langle\tilde{m}_{l}\right\rangle_{\mathrm{LR}}^{2}} \frac{10}{\tan \beta}\left(\frac{\bar{\varepsilon}}{0.13}\right)^{3} \\
& \left|y_{1}\right|\left|x_{123}-x_{23}-x_{\Sigma}\right| .
\end{aligned}
$$

where $\bar{\epsilon}$ is the expansion parameter determining the mixing in the down quark charged lepton sector [225] (of the order of the Cabibbo angle) and $y_{1}$ and $x_{123,23, \Sigma}$ are parameters that are typically of order 1 . The parameter $y_{1}$ is the coefficient of the leading super potential term generating the lepton mixing and $x_{123,23, \Sigma}$ are the coefficients multiplying the natural magnitudes of the $\mathrm{F}$ terms of the familon fields. For the $\mathrm{EDM}$ one finds for the relevant mass insertion parameters [218-220]

$$
\begin{aligned}
&\left|\operatorname{Im}\left(\delta_{\mathrm{LR}}^{u}\right)_{11}\right| \approx 2 \times 10^{-7} \frac{A_{0}}{100 \mathrm{GeV}}\left(\frac{500 \mathrm{GeV}}{\left\langle\tilde{m}_{u}\right\rangle_{\mathrm{LR}}}\right)^{2}\left(\frac{\bar{\varepsilon}}{0.13}\right)^{3}\left(\frac{\varepsilon}{0.05}\right)^{2} \\
&\left|y_{1}^{f}+y_{2}^{f}\right|\left|x_{123}-x_{23}-x_{\Sigma}\right| \sin \phi_{1}, \\
&\left|\operatorname{Im}\left(\delta_{\mathrm{LR}}^{d}\right)_{11}\right| \approx 5 \times 10^{-7} \frac{A_{0}}{100 \mathrm{GeV}}\left(\frac{500 \mathrm{GeV}}{\left\langle\tilde{m}_{d}\right\rangle_{\mathrm{LR}}}\right)^{2}\left(\frac{\bar{\varepsilon}}{0.13}\right)^{5} \frac{10}{\tan \beta} \\
&\left|y_{1}^{f}+y_{2}^{f}\right|\left|x_{123}-x_{23}-x_{\Sigma}\right| \sin \phi_{1},
\end{aligned}
$$

\footnotetext{
${ }^{13}$ For a more general analysis and comparison with MFV expectations, see [221].
} 


$$
\begin{aligned}
\left|\operatorname{Im}\left(\delta_{\mathrm{LR}}^{\ell}\right)_{11}\right| \approx & 2 \times 10^{-7} \frac{A_{0}}{100 \mathrm{GeV}}\left(\frac{200 \mathrm{GeV}}{\left\langle\tilde{m}_{e}\right\rangle_{\mathrm{LR}}}\right)^{2}\left(\frac{\bar{\varepsilon}}{0.13}\right)^{5} \frac{10}{\tan \beta} \\
& \left|y_{1}^{f}+y_{2}^{f}\right|\left|x_{123}-x_{23}-x_{\Sigma}\right| \sin \phi_{1},
\end{aligned}
$$

where $\epsilon$ is the expansion parameter determining the mixing in the up quark sector, $y_{i}^{f}$ are the analogues of $y_{1}$ in the quark sector and $\phi_{1}$ is a CP phase associated with the VEV of the relevant familon field.

The present experimental bound from the non-observation of $\mu \rightarrow e \gamma$ is $\left|\left(\delta_{\mathrm{LR}}^{\ell}\right)_{12}\right| \leq 10^{-5}$, which is in some tension with this bound, requiring, for example, $\tilde{m}_{l}=600 \mathrm{GeV}$ if the remaining factors in Eq. (33) are of $O(1)$. For the EDMs the most stringent bound comes from mercury and corresponds to $\left|\operatorname{Im}\left(\delta_{\mathrm{LR}}^{d}\right)_{11}\right|<6.7 \times 10^{-8}$ and requires $\tilde{m}_{d}=1,500 \mathrm{GeV}$ if the other factors are of $O(1)$.

It is interesting that the SUSY mass scales needed for consistency with the FCNC and CP-violating bounds are close to the increased mass scales needed to accommodate the $125 \mathrm{GeV}$ Higgs mass, suggesting that the experimental limits on $\mathrm{CP}$ and FCNC may be quite close to the actual rates ${ }^{14}$.

\section{Summary and conclusions}

The discovery of a Higgs candidate at $126 \mathrm{GeV}$ may be seen as a success for the idea that there is a low-scale SUSY extension of the SM because the Higgs mass lies in the relatively small range favoured by SUSY. Moreover the observed properties of the candidate Higgs are just what are expected of an elementary state whose interactions are in the perturbative domain, as is expected in SUSY with gauge coupling unification. However, there is no doubt that evidence for SUSY is overdue in the sense that the naive expectation is that there should be SUSY partners of SM states with EW scale masses, accessible at LEP. The non-observation, to date, of coloured SUSY states at the LHC has made this discrepancy considerably worse as such states are pushed up to the TeV scale. This discrepancy is exacerbated by the Higgs discovery, as its mass lies at the upper end of the anticipated range and, at least in simple models, requires large radiative corrections to the Higgs mass and the corresponding need for large SUSY breaking parameters. Fits to the simple models, the CMSSM and the CNMSSM, show that they are under considerable pressure with only a very small region of parameter space consistent will all available data including the dark matter abundance and direct dark matter searches.

To sharpen the test of SUSY as a solution to the (little) hierarchy problem it is necessary to quantify the level of fine

\footnotetext{
14 This depends on the $O(1)$ assumption for the values of the $x, y$ parameters. While this is the most natural value there is a mechanism capable of suppressing the rates a bit more, by an extra power of $\varepsilon$ in $\left|\operatorname{Im}\left(\delta_{\mathrm{LR}}^{u}\right)_{11}\right|$ and of $\bar{\varepsilon}$ in $\left|\operatorname{Im}\left(\delta_{\mathrm{LR}}^{d}\right)_{11}\right|$ and $\left|\operatorname{Im}\left(\delta_{\mathrm{LR}}^{l}\right)_{11}\right|$, respectively (for further details see [220]).
}

tuning needed to generate the observed EW scale in the presence of TeV-scale SUSY. Recent work has shown that, for the case that the EW breaking scale is treated as a nuisance variable, the fine tuning measures of fine tuning commonly used can be derived from a likelihood fit of the SUSY model to the present data. The advantage of this is that one can put an upper bound on the fine tuning consistent with an acceptable fit to the data. Applying this to simple SUSY extensions of the SM, namely the CMSSM and the CNMSSM, gives unacceptably large values of fine tuning, confirming the naive expectation. However, this does not mean that the possibility that SUSY solves the little hierarchy problem has already been excluded because it may be that non-minimal implementations of SUSY are not heavily fine-tuned. Fine tuning is strongly dependent both on the nature of the soft SUSY breaking terms and on the spectrum of the particular SUSY extension of the SM and both possibilities have been explored.

As is illustrated by the scalar focus point in the CMSSM and CNMSSM that results from the assumption of degenerate scalars at the initial (unification) scale, correlations between the soft parameters can reduce the fine-tuning substantially. The only other theoretically well-motivated focus point that has been found corresponds to gaugino masses that are nondegenerate at the initial scae so that the gluino and winos are close in mass at the EW scale. Modifying the initial conditions for gaugino masses in the CMSSM and CNMSSM to achieve this does result in a considerable reduction in fine tuning but it is still uncomfortably large.

The effect of a new states beyond those of the MSSM has, for the case these states are very heavy, been explored in a model independent way via the introduction of higher dimension operators. This shows that, even for the mass of the additional states in the multi $\mathrm{TeV}$ region, the fine tuning can be substantially reduced, largely by generating an additional contribution to the Higgs mass, reducing the need for very large SUSY breaking terms. The simplest way to generate the operator most significant for the reduction in fine tuning requires the addition of a singlet super field to the MSSM, as in the NMSSM. However, it requires a generalisation of the usual NMSSM through the addition of explicit EW scale mass terms for the Higgsinos and the singlet, something that can be ensured by a discrete R-symmetry. Analysis of this model shows that with the normal constrained initial spectrum the fine tuning is still uncomfortably large but allowing for non-universal gaugino masses there is a substantial region of the parameter space for which the fine-tuning drops to perfectly acceptable level even for squarks and the gluino with masses in the (1-3) TeV region. Indeed the fine tuning is close to the minimum found for the variation of the parameters at a low (EW) scale only, showing that it is possible to achieve such low fine tuning in a UV complete theory that preserves the success of gauge coupling unification. The large mass of 
the coloured SUSY states, together with the fact that often the low fine tuned spectra are considerable compressed, corresponding to low $E_{T}$ missing signals, means that it may be difficult to test the model fully even at LHC14. Dark matter can be consistent with the WMAP bounds and direct detection limits, the expectation of all the models analysed being that the LSP should be heavy, of $O(1 \mathrm{TeV})$, and most often with a substantial Higgsino component. However, the scattering cross section can be very low so that the low fine tuned region will not be fully tested even by XENON1T.

An associated effect of the increased bound on the SUSY masses is that the constraints from flavour-changing neutral currents and CP violation are significantly reduced. It is still necessary that the new $\mathrm{CP}$-violating phases associated with the flavour singlet sector be small but this can be readily done in models with an underlying family symmetry. However, this provides only a relatively modest suppression and the expectation is that processes such as $\mu \rightarrow e \gamma$ and electric dipole moments will be close to their present limits.

Open Access This article is distributed under the terms of the Creative Commons Attribution License which permits any use, distribution, and reproduction in any medium, provided the original author(s) and the source are credited.

Funded by $\mathrm{SCOAP}^{3}$ / License Version CC BY 4.0.

\section{References}

1. G. Aad et al., ATLAS Collaboration, Phys. Lett. B 716, 1 (2012). [arXiv:1207.7214 [hep-ex]]

2. S. Chatrchyan et al., CMS Collaboration, Phys. Lett. B 716, 30 (2012). [arXiv:1207.7235 [hep-ex]]

3. V.D. Barger, M.S. Berger, P. Ohmann, Phys. Rev. D 49, 4908 (1994). [arXiv:hep-ph/9311269]

4. W. de Boer, R. Ehret, D.I. Kazakov, Z. Phys, C 67, 647 (1995). [arXiv:hep-ph/9405342]

5. M. Carena, J.R. Ellis, A. Pilaftsis, C.E. Wagner, Nucl. Phys. B 625, 345 (2002). [arXiv:hep-ph/0111245]

6. H.E. Haber, R. Hempfling, Phys. Rev. Lett. 66, 1815 (1991)

7. J.R. Ellis, G. Ridolfi, F. Zwirner, Phys. Lett. B 257, 83 (1991)

8. Y. Okada, M. Yamaguchi, T. Yanagida, Prog. Theor. Phys. 85, 1 (1991)

9. M. Drees, M.M. Nojiri, Phys. Rev. D 47, 376 (1993). [arXiv: hep-ph/9207234]

10. H. Baer, M. Brhlik, Phys. Rev. D 53, 597 (1996). [arXiv:hep-ph/ 9508321]

11. H. Baer, M. Brhlik, Phys. Rev. D 57, 567 (1998). [arXiv:hep-ph/ 9706509]

12. H. Baer, M. Brhlik, M.A. Diaz, J. Ferrandis, P. Mercadante, P. Quintana, X. Tata, Phys. Rev. D 63, 015007 (2001). [arXiv: hep-ph/0005027]

13. G.L. Kane, C.F. Kolda, L. Roszkowski, J.D. Wells, Phys. Rev. D 49, 6173 (1994). [arXiv:hep-ph/9312272]

14. J.R. Ellis, T. Falk, K.A. Olive, M. Schmitt, Phys. Lett. B 388, 97 (1996). [arXiv:hep-ph/9607292]

15. J.R. Ellis, T. Falk, K.A. Olive, M. Schmitt, Phys. Lett. B 413, 355 (1997). [arXiv:hep-ph/9705444]
16. J.R. Ellis, T. Falk, G. Ganis, K.A. Olive, M. Schmitt, Phys. Rev. D 58, 095002 (1998). [arXiv:hep-ph/9801445]

17. V.D. Barger, C. Kao, Phys. Rev. D 57, 3131 (1998). [arXiv: hep-ph/9704403]

18. J.R. Ellis, T. Falk, G. Ganis, K.A. Olive, Phys. Rev. D 62, 075010 (2000). [arXiv:hep-ph/0004169]

19. J.R. Ellis, T. Falk, G. Ganis, K.A. Olive, M. Srednicki, Phys. Lett. B 510, 236 (2001). [arXiv:hep-ph/0102098]

20. V.D. Barger, C. Kao, Phys. Lett. B 518, 117 (2001). [arXiv: hep-ph/0106189]

21. L. Roszkowski, R. Ruiz de Austri, T. Nihei, JHEP 0108, 024 (2001). [arXiv:hep-ph/0106334]

22. A. Djouadi, M. Drees, J.L. Kneur, JHEP 0108, 055 (2001). [arXiv: hep-ph/0107316]

23. U. Chattopadhyay, A. Corsetti, P. Nath, Phys. Rev. D 66, 035003 (2002). [arXiv:hep-ph/0201001]

24. J.R. Ellis, K.A. Olive, Y. Santoso, New J. Phys. 4, 32 (2002). [arXiv:hep-ph/0202110]

25. H. Baer, C. Balazs, A. Belyaev, J.K. Mizukoshi, X. Tata, Y. Wang, JHEP 0207, 050 (2002). [arXiv:hep-ph/0205325]

26. R. Arnowitt, B. Dutta [arXiv:hep-ph/0211417]

27. J.R. Ellis, K.A. Olive, Y. Santoso, V.C. Spanos, Phys. Lett. B 565, 176 (2003). [arXiv:hep-ph/0303043]

28. H. Baer, C. Balazs, JCAP 0305, 006 (2003). [arXiv:hep-ph/ 0303114]

29. A.B. Lahanas, D.V. Nanopoulos, Phys. Lett. B 568, 55 (2003). [arXiv:hep-ph/0303130]

30. U. Chattopadhyay, A. Corsetti, P. Nath, Phys. Rev. D 68, 035005 (2003). [arXiv:hep-ph/0303201]

31. C. Munoz, Int. J. Mod. Phys. A 19, 3093 (2004). [arXiv:hep-ph/ 0309346]

32. R. Arnowitt, B. Dutta, B. Hu [arXiv:hep-ph/0310103]

33. J. Ellis, K. A. Olive, in Particle dark matter, ed. by G. Bertone, pp. 142-163 [arXiv:1001.3651 [astro-ph.CO]]

34. O. Buchmueller et al. [arXiv:1207.7315 [hep-ph]]

35. C. Strege, G. Bertone, D.G. Cerdeno, M. Fornasa, R.R. de Austri, R. Trotta, JCAP 1203, 030 (2012). [arXiv:1112.4192 [hep-ph]]

36. P. Bechtle, T. Bringmann, K. Desch, H. Dreiner, M. Hamer, C. Hensel, M. Kramer, N. Nguyen et al., JHEP 1206, 098 (2012). [arXiv:1204.4199 [hep-ph]]

37. A. Fowlie, M. Kazana, K. Kowalska, S. Munir, L. Roszkowski, E. M. Sessolo, S. Trojanowski, Y. -L. S. Tsai [arXiv:1206.0264 [hep-ph]]

38. T. Li, J. A. Maxin, D. V. Nanopoulos, J. W. Walker [arXiv:1206. 2633 [hep-ph]]

39. O. Buchmueller et al., Eur. Phys. J. C 72, 2020 (2012). [arXiv: 1112.3564 [hep-ph]]

40. H. Baer, V. Barger, A. Mustafayev, Phys. Rev. D 85, 075010 (2012). [arXiv:1112.3017 [hep-ph]]

41. J.L. Feng, K.T. Matchev, D. Sanford, Phys. Rev. D 85, 075007 (2012). [arXiv:1112.3021 [hep-ph]]

42. T. Li, J.A. Maxin, D.V. Nanopoulos, J.W. Walker, Phys. Lett. B 710, 207 (2012). [arXiv:1112.3024 [hep-ph]]

43. S. Heinemeyer, O. Stal, G. Weiglein, Phys. Lett. B 710, 201 (2012). [arXiv:1112.3026 [hep-ph]]

44. A. Arbey, M. Battaglia, A. Djouadi, F. Mahmoudi, J. Quevillon, Phys. Lett. B 708, 162 (2012). [arXiv:1112.3028 [hep-ph]]

45. P. Draper, P. Meade, M. Reece, D. Shih, Phys. Rev. D 85, 095007 (2012). [arXiv:1112.3068 [hep-ph]]

46. S. Akula, B. Altunkaynak, D. Feldman, P. Nath, G. Peim, Phys. Rev. D 85, 075001 (2012). [arXiv:1112.3645 [hep-ph]]

47. M. Kadastik, K. Kannike, A. Racioppi, M. Raidal, JHEP 1205, 061 (2012). [arXiv:1112.3647 [hep-ph]]

48. J. Cao, Z. Heng, D. Li, J.M. Yang, Phys. Lett. B 710, 665 (2012). [arXiv:1112.4391 [hep-ph]] 
49. L. Aparicio, D.G. Cerdeno, L.E. Ibanez, JHEP 1204, 126 (2012). [arXiv:1202.0822 [hep-ph]]

50. H. Baer, V. Barger, A. Mustafayev, JHEP 1205, 091 (2012). [arXiv:1202.4038 [hep-ph]]

51. C. Balazs, A. Buckley, D. Carter, B. Farmer, M. White [arXiv: 1205.1568 [hep-ph]]

52. D. Ghosh, M. Guchait, S. Raychaudhuri, D. Sengupta, Phys. Rev. D 86, 055007 (2012). [arXiv:1205.2283 [hep-ph]]

53. J. Ellis, K.A. Olive, Eur. Phys. J. C 72, 2005 (2012). [arXiv:1202. 3262 [hep-ph]]

54. J. Ellis, F. Luo, K.A. Olive, P. Sandick, Eur. Phys. J. C 73, 2403 (2013). [arXiv:1212.4476 [hep-ph]]

55. A. Djouadi, L. Maiani, G. Moreau, A. Polosa, J. Quevillon, V. Riquer [arXiv:1307.5205 [hep-ph]]

56. C. Beskidt, W. de Boer, D. I. Kazakov [arXiv:1308.1333 [hep-ph]]

57. S. Zheng, Y. Yu, JHEP 1308, 031 (2013). [arXiv:1303.1900 [hep$\mathrm{ph}]]$

58. P. Bechtle, S. Heinemeyer, O. Stal, T. Stefaniak, G. Weiglein, L. Zeune, Eur. Phys. J. C 73, 2354 (2013). [arXiv:1211.1955 [hep$\mathrm{ph}]]$

59. M. Kramer, P. Bechtle, K. Desch, H.K. Dreiner, B. O'Leary, C. Robens, B. Sarrazin, P. Wienemann, Frascati Phys. Ser. 54, 258 (2012)

60. ATLAS Collaboration. https://cdsweb.cern.ch/record/1432199/ files/ATLAS-CONF-2012-033.pdf; https://cdsweb.cern.ch/ record/1472710/files/ATLAS-CONF-2012-109.pdf

61. CMS Collaboration, S. Chatrchyan et al., JHEP 1210, 018 (2012). [arXiv:1207.1798 [hep-ex]]; [arXiv:1207.1898 [hep-ex]]

62. E. Komatsu et al., WMAP Collaboration, Astrophys. J. Suppl. 192, 18 (2011). [arXiv:1001.4538 [astro-ph.CO]]

63. E. Aprile et al., XENON100 Collaboration, Phys. Rev. Lett. 107, 131302 (2011). [arXiv:1104.2549 [astro-ph.CO]]

64. S. Chen et al., CLEO Collaboration, Phys. Rev. Lett. 87, 251807 (2001). [arXiv:hep-ex/0108032]

65. P. Koppenburg et al., Belle Collaboration, Phys. Rev. Lett. 93, 061803 (2004). [arXiv:hep-ex/0403004]

66. B. Aubert et al., BaBar Collaboration [arXiv:hep-ex/0207076]

67. E. Barberio et al., Heavy Flavor Averaging, Group (HFAG) [arXiv: hep-ex/0603003]

68. G. Aad et al., ATLAS Collaboration, Phys. Lett. B 713, 387 (2012). [arXiv:1204.0735 [hep-ex]]

69. T. Aaltonen et al., CDF Collaboration, Phys. Rev. Lett. 107, 239903 (2011). [Phys. Rev. Lett. 107, 191801 (2011)] [arXiv: 1107.2304 [hep-ex]]

70. S. Chatrchyan et al., CMS Collaboration, Phys. Rev. Lett. 107, 191802 (2011). [arXiv:1107.5834 [hep-ex]]

71. R. Aaij et al., LHCb Collaboration, Phys. Lett. B 699, 330 (2011). [arXiv:1103.2465 [hep-ex]]; [arXiv:1203.4493 [hep-ex]]

72. For an official combination of the ATLAS, CMS and LHCb results, see: ATLAS, CMS, and LHCb Collaborations. http:// cdsweb.cern.ch/record/1452186/files/LHCb-CONF-2012-017. pdf

73. LHCb Collaboration, R. Aaij et al., Phys. Rev. Lett. 110, 021801 (2013). [arXiv:1211.2674 [Unknown]]

74. The Muon g-2 Collaboration, Phys. Rev. Lett. 92, 161802 (2004). [hep-ex/0401008]

75. G. Bennett et al., The Muon g-2 Collaboration. Phys. Rev. D 73, 072003 (2006). [arXiv:hep-ex/0602035]

76. G. Abbiendi et al., OPAL Collaboration. Eur. Phys. J. C35, 1-20 (2004). [hep-ex/0401026]

77. A. Fowlie, M. Kazana, K. Kowalska, S. Munir, L. Roszkowski, E.M. Sessolo, S. Trojanowski, Y.-L.S. Tsai, Phys. Rev. D 86, 075010 (2012). [arXiv:1206.0264 [hep-ph]]

78. C. Strege, G. Bertone, F. Feroz, M. Fornasa, R. Ruiz de Austri, R. Trotta, JCAP 1304, 013 (2013). [arXiv:1212.2636 [hep-ph]]
79. Information about this code is available from K. A. Olive: it contains important contributions from T. Falk, A. Ferstl, F. Luo, G. Ganis, A. Mustafayev, J. McDonald, K. A. Olive, P. Sandick, Y. Santoso, V. Spanos, and M. Srednicki

80. H. Baer, A. Mustafayev, S. Profumo, A. Belyaev, X. Tata, Phys. Rev. D 71, 095008 (2005). [arXiv:hep-ph/0412059]

81. H. Baer, A. Mustafayev, S. Profumo, A. Belyaev, X. Tata, JHEP 0507, 065 (2005). [hep-ph/0504001]

82. J.R. Ellis, K.A. Olive, P. Sandick, Phys. Rev. D 78, 075012 (2008), [arXiv:0805.2343 [hep-ph]]

83. J. Ellis, K. Olive, Y. Santoso, Phys. Lett. B 539, 107 (2002). [arXiv: hep-ph/0204192]

84. J.R. Ellis, T. Falk, K.A. Olive, Y. Santoso, Nucl. Phys. B 652, 259 (2003). [arXiv:hep-ph/0210205]

85. D. Matalliotakis, H.P. Nilles, Nucl. Phys. B 435, 115 (1995). [arXiv:hep-ph/9407251]

86. M. Olechowski, S. Pokorski, Phys. Lett. B 344, 201 (1995). [arXiv:hep-ph/9407404]

87. V. Berezinsky, A. Bottino, J. Ellis, N. Fornengo, G. Mignola, S. Scopel, Astropart. Phys. 5, 1 (1996). [hep-ph/9508249]

88. M. Drees, M. Nojiri, D. Roy, Y. Yamada, Phys. Rev. D 56, 276 (1997) [Erratum-ibid. D 64 (1997) 039901] [hep-ph/9701219]

89. M. Drees, Y. Kim, M. Nojiri, D. Toya, K. Hasuko, T. Kobayashi, Phys. Rev. D 63, 035008 (2001). [hep-ph/0007202]

90. P. Nath, R. Arnowitt, Phys. Rev. D 56, 2820 (1997). [hep-ph/ 9701301]

91. A. Bottino, F. Donato, N. Fornengo, S. Scopel, Phys. Rev. D 63, 125003 (2001). [hep-ph/0010203]

92. S. Profumo, Phys. Rev. D 68, 015006 (2003). [hep-ph/0304071]

93. D. Cerdeno, C. Munoz, JHEP 0410, 015 (2004). [hep-ph/ 0405057]

94. U. Ellwanger, C. Hugonie, A. M. Teixeira, Phys. Rep. 496, 1-77 (2010). [arXiv:0910.1785 [hep-ph]]

95. M. Maniatis, Int. J. Mod. Phys. A25, 3505-3602 (2010). [arXiv: 0906.0777 [hep-ph]]

96. U. Ellwanger, JHEP 1203, 044 (2012). [arXiv:1112.3548 [hep$\mathrm{ph}]]$

97. U. Ellwanger, C. Hugonie, Adv. High Energy Phys. 2012, 625389 (2012). [arXiv:1203.5048 [hep-ph]]

98. S. King, M. Muhlleitner, R. Nevzorov, Nucl. Phys. B 860, 207244 (2012). [arXiv:1201.2671 [hep-ph]]

99. J. Cao et al., JHEP 1203, 086 (2012). [arXiv:1202.5821 [hep-ph]]

100. D.A. Vasquez, G. Belanger, C. Boehm, J. Da Silva, P. Richardson et al., Phys. Rev. D 86, 035023 (2012). [arXiv:1203.3446 [hep$\mathrm{ph}]]$

101. J. Rathsman, T. Rossler, Adv. High Energy Phys. 2012, 853706 (2012). [arXiv:1206.1470 [hep-ph]]

102. D. Das, U. Ellwanger, P. Mitropoulos, JCAP 1208, 003 (2012). [arXiv:1206.2639 [hep-ph]]

103. J.F. Gunion, Y. Jiang, S. Kraml, Phys. Rev. D 86, 071702 (2012). [arXiv:1207.1545 [hep-ph]]

104. J.F. Gunion, Y. Jiang, S. Kraml, Phys. Rev. Lett. 110, 051801 (2013). [arXiv:1208.1817 [hep-ph]]

105. R. Benbrik, M. Gomez, Bock, S. Heinemeyer, O. Stal, G. Weiglein, et al., Eur. Phys. J. C72, 2171 (2012). [arXiv:1207.1096 [hep-ph]]

106. K. J. Bae, K. Choi, E. J. Chun, S. H. Im, C. B. Park, et al., JHEP 1211, 118 (2012). [arXiv:1208.2555 [hep-ph]]

107. Z. Kang, T. Li, J. Li, Y. Liu [arXiv:1208.2673 [hep-ph]]

108. T. Cheng, J. Li, T. Li, X. Wan, Y. K. Wang et al. [arXiv:1207.6392 [hep-ph]]

109. M. Perelstein, B. Shakya [arXiv:1208.0833 [hep-ph]]

110. G. Belanger, U. Ellwanger, J. Gunion, Y. Jiang, S. Kraml [arXiv: 1208.4952 [hep-ph]]

111. J. Cao, Z. Heng, J. M. Yang, J. Zhu, JHEP 1210, 079 (2012). [arXiv:1207.3698 [hep-ph]] 
112. G. Chalons, F. Domingo, Phys. Rev. D 86, 115024 (2012). [arXiv: 1209.6235 [hep-ph]]

113. G. Belanger, U. Ellwanger, J. F. Gunion, Y. Jiang, S. Kraml, et al., JHEP 1301, 069 (2013). [arXiv:1210.1976 [hep-ph]]

114. I. Gogoladze, B. He, Q. Shafi, Phys. Lett. B 718, 1008-1013 (2013). [arXiv:1209.5984 [hep-ph]]

115. D.E. Lopez-Fogliani, J. Phys. Conf. Ser. 384, 012014 (2012)

116. J. F. Gunion, Y. Jiang, S. Kraml, Phys. Lett. B 710, 454-459 (2012). [arXiv:1201.0982 [hep-ph]]

117. J. Cao, F. Ding, C. Han, J. M. Yang, J. Zhu [arXiv:1309.4939 [hep-ph]]

118. K. Kowalska, S. Munir, L. Roszkowski, E.M. Sessolo, S. Trojanowski, Y.-L.S. Tsai, Phys. Rev. D 87, 115010 (2013). [arXiv: 1211.1693 [hep-ph]]

119. G.L. Kane, S.F. King, Phys. Lett. B 451, 113 (1999). [hep-ph/ 9810374]

120. K.L. Chan, U. Chattopadhyay, P. Nath, Phys. Rev. D 58, 096004 (1998). [arXiv:hep-ph/9710473]

121. J.L. Feng, K.T. Matchev, T. Moroi, Phys. Rev. Lett. 84, 2322 (2000). [hep-ph/9908309]

122. J.L. Feng, K.T. Matchev, T. Moroi, Phys. Rev. D 61, 075005 (2000). [hep-ph/9909334]

123. J.L. Feng, K.T. Matchev, F. Wilczek, Phys. Rev. D 63, 045024 (2001). [astro-ph/0008115]

124. J.L. Feng, K.T. Matchev, D. Sanford, Phys. Rev. D 85, 075007 (2012). [arXiv:1112.3021 [hep-ph]]

125. J.R. Ellis, K. Enqvist, D.V. Nanopoulos, F. Zwirner, Mod. Phys. Lett. A1, 57 (1986)

126. R. Barbieri, G.F. Giudice, Nucl. Phys. B 306, 63 (1988)

127. D.M. Ghilencea, G.G. Ross, Nucl. Phys. B 868, 65 (2013). [arXiv: 1208.0837 [hep-ph]]

128. D. M. Ghilencea [arXiv:1302.5262 [hep-ph]]

129. PoS Corfu 2012, 034 (2013). [arXiv:1304.1193 [hep-ph]]

130. M.E. Cabrera, J.A. Casas, R. Ruiz de Austri, JHEP 0903, 075 (2009). [arXiv:0812.0536 [hep-ph]]

131. C.H. Llewellyn Smith, G. G. Ross. Phys. Lett. B 105, 38 (1981)

132. G. Marques Tavares, M. Schmaltz, W. Skiba [arXiv:1308.0025 [hep-ph]]

133. H. Baer, V. Barger, P. Huang, D. Mickelson, A. Mustafayev, W. Sreethawong, X. Tata [arXiv:1306.3148 [hep-ph]]

134. H. Baer, V. Barger, P. Huang, A. Mustafayev, X. Tata, Phys. Rev. Lett. 109, 161802 (2012). [arXiv:1207.3343 [hep-ph]]

135. H. Baer, V. Barger, P. Huang, D. Mickelson, A. Mustafayev, X. Tata, Phys. Rev. D 87, 115028 (2013). [arXiv:1212.2655 [hep$\mathrm{ph}]]$

136. H. Baer, V. Barger, P. Huang, D. Mickelson, A. Mustafayev, X. Tata [arXiv:1306.2926 [hep-ph]]

137. D.M. Ghilencea, H.M. Lee, M. Park, JHEP 1207, 046 (2012). [arXiv:1203.0569 [hep-ph]]

138. S. Cassel, D.M. Ghilencea, S. Kraml, A. Lessa, G.G. Ross, JHEP 1105, 120 (2011). [arXiv:1101.4664 [hep-ph]]

139. S. Abel, M.J. Dolan, J. Jaeckel, V.V. Khoze, JHEP 1012, 049 (2010). [arXiv:1009.1164 [hep-ph]]

140. S. Abel, M.J. Dolan, J. Jaeckel, V.V. Khoze, JHEP 0912, 001 (2009). [arXiv:0910.2674 [hep-ph]]

141. S. Dimopoulos, G. Giudice, Phys. Lett. B 357, 573-578 (1995). [hep-ph/9507282]

142. A.G. Cohen, D. Kaplan, A. Nelson, Phys. Lett. B 388, 588-598 (1996). [hep-ph/9607394]

143. E. Hardy [arXiv:1306.1534 [hep-ph]]

144. A. Arvanitaki, M. Baryakhtar, X. Huang, K. Van Tilburg, G. Villadoro [arXiv:1309.3568 [hep-ph]]

145. D. Horton, G. Ross, Nucl. Phys. B830 (2010), 221-247, [0908.0857] K. Choi, K. S. Jeong, T. Kobayashi, and K.-I. Okumura. Phys. Lett. B 633, 355-361 (2006). [hep-ph/0508029]
146. K. Choi, K.S. Jeong, T. Kobayashi, K.-I. Okumura. Phys. Lett. B 633, 355-361 (2006). [hep-ph/0508029]

147. K. Choi, K.S. Jeong, T. Kobayashi, K.-I. Okumura, Phys. Rev. D 75, 095012 (2007). [hep-ph/0612258]

148. H. Abe, T. Kobayashi, Y. Omura, Phys. Rev. D 76, 015002 (2007). [hep-ph/0703044]

149. O. Lebedev, H.P. Nilles, M. Ratz, (2005), 211-221, hepph/0511320 (M. Asano and T, Higaki, 2012). 1204.0508

150. M. Asano, T. Higaki (2012). [1204.0508]

151. S. Antusch, L. Calibbi, V. Maurer, M. Monaco, M. Spinrath, JHEP 01, 187 (2013). [1207.7236]

152. H. Abe, J. Kawamura, H. Otsuka, PTEP 2013, $013 B 02$ (2013). [1208.5328]

153. M. Badziak, S. Krippendorf, H.P. Nilles, M.W. Winkler, JHEP 1303, 094 (2013). [1212.0854]

154. I. Gogoladze, F. Nasir, Q. Shafi, Int. J. Mod. Phys. A 28, 1350046 (2013). [1212.2593]

155. T.T. Yanagida, N. Yokozaki, Phys. Lett. B 722, 355-359 (2013). [1301.1137]

156. L. Roszkowski, M.A. Shifman, Phys. Rev. D 53, 404 (1996). [hep-ph/9503358]

157. S.P. Martin, Phys. Rev. D 79, 095019 (2009). [0903.3568]

158. A. Brignole, L.E. Ibanez, C. Munoz, Nucl. Phys. B 422, 125-171 (1994). [hep-ph/9308271]

159. K. Choi, K.S. Jeong, T. Kobayashi, K.-I. Okumura, Phys. Lett. B 633, 355-361 (2006). [hep-ph/0508029]

160. O. Lebedev, H. P. Nilles, M. Ratz, 211-221 (2005). [hep-ph/ 0511320]

161. K. Choi, H.P. Nilles, JHEP 0704, 006 (2007). [hep-ph/0702146]

162. W. Cho, Y. Kim, K. Lee, C. Park, Y. Shimizu, JHEP 0704, 054 (2007). [hep-ph/0703163]

163. K. Choi, K.S. Jeong, K.-I. Okumura, JHEP 0509, 039 (2005). [hep-ph/0504037]

164. M. Badziak, S. Krippendorf, H.P. Nilles, M.W. Winkler, JHEP 1303, 094 (2013). [1212.0854]

165. S. Antusch, L. Calibbi, V. Maurer, M. Monaco, M. Spinrath, JHEP 01, 187 (2013). [1207.7236]

166. I. Antoniadis, E. Dudas, D.M. Ghilencea, P. Tziveloglou, Nucl. Phys. B 848, 1 (2011). [arXiv: 1012.5310 [hep-ph]]

167. I. Antoniadis, E. Dudas, D.M. Ghilencea, P. Tziveloglou, Nucl. Phys. B 831, 133 (2010). [arXiv:0910.1100 [hep-ph]]

168. I. Antoniadis, E. Dudas, D.M. Ghilencea, P. Tziveloglou, Nucl. Phys. B 808, 155 (2009). [arXiv:0806.3778 [hep-ph]]

169. D. Piriz, J. Wudka, Phys. Rev. D 56, 4170 (1997). [hep-ph/ 9707314]

170. S.P. Martin, Phys. Rev. D 61, 035004 (2000). [hep-ph/9907550]

171. R. Barbieri, A. Strumia, Phys. Lett. B 462, 144 (1999). [hep-ph/ 9905281]

172. M. Dine, N. Seiberg, S. Thomas, Phys. Rev. D 76, 095004 (2007). [arXiv:0707.0005 [hep-ph]]

173. J. A. Casas, J. R. Espinosa. I. Hidalgo [hep-ph/0402017]

174. S. Cassel, D.M. Ghilencea, G.G. Ross, Nucl. Phys. B 825, 203 221 (2010). [0903.1115]

175. X. Lu, H. Murayama, J. T. Ruderman, K. Tobioka [arXiv:1308. 0792 [hep-ph]]

176. H.M. Lee, S. Raby, M. Ratz, G.G. Ross, R. Schieren, K. SchmidtHoberg, P.K.S. Vaudrevange, Phys. Lett. B 694, 491 (2011). [arXiv:1009.0905 [hep-ph]]

177. H.M. Lee, S. Raby, M. Ratz, G.G. Ross, R. Schieren, K. SchmidtHoberg, P.K.S. Vaudrevange, Nucl. Phys. B 850, 1 (2011). [arXiv: 1102.3595 [hep-ph]]

178. G.G. Ross, K. Schmidt-Hoberg, Nucl. Phys. B 862, 710 (2012). [arXiv:1108.1284 [hep-ph]]

179. G. G. Ross, K. Schmidt-Hoberg, F. Staub, JHEP 1208, 074 (2012). [arXiv:1205.1509 [hep-ph]] 
180. A. Delgado, C. Kolda, A. de la Puente, Phys. Lett. B 710, 460 (2012). [arXiv:1111.4008 [hep-ph]]

181. A. Delgado, C. Kolda, J.P. Olson, A. de la Puente, Phys. Rev. Lett. 105, 091802 (2010). [arXiv:1005.1282 [hep-ph]]

182. A. Delgado, C. Kolda, J.P. Olson, A. de la Puente, Phys. Rev. D 82, 035006 (2010). [arXiv:1005.4901 [hep-ph]]

183. L.J. Hall, D. Pinner, J.T. Ruderman, JHEP 1204, 131 (2012). [arXiv:1112.2703 [hep-ph]]

184. A. Kaminska, G. G. Ross, K. Schmidt-Hoberg [arXiv:1308.4168 [hep-ph]]

185. L. Roszkowski, R. Ruiz de Austri, R. Trotta, Y.-L.S. Tsai, T.A. Varley, Phys. Rev. D 83, 015014 (2011). [arXiv:0903.1279 [hep$\mathrm{ph}]]$

186. S. Akula, P. Nath, G. Peim, Phys. Lett. B 717, 188 (2012). [arXiv: 1207.1839 [hep-ph]]

187. T.J. LeCompte, S.P. Martin, Phys. Rev. D 84, 015004 (2011). [arXiv:1105.4304 [hep-ph]]

188. H.K. Dreiner, M. Kramer, J. Tattersall, Europhys. Lett. 99, 61001 (2012). [arXiv:1207.1613 [hep-ph]]

189. L.E. Ibanez, G.G. Ross, Nucl. Phys. B 368, 3 (1992)

190. H.K. Dreiner, C. Luhn, M. Thormeier, Phys. Rev. D 73, 075007 (2006). [hep-ph/0512163]

191. J. A. Evans, Y. Kats [arXiv:1209.0764]

192. Z. Han, A. Katz, M. Son, B. Tweedie [arXiv:1211.4025]

193. B. Allanach, B. Gripaios, JHEP 1205, 062 (2012). [arXiv:1202. 6616]

194. C. Brust, A. Katz, R. Sundrum, JHEP 1208, 059 (2012). [arXiv: 1206.2353]

195. CMS Collaboration Collaboration, S. Chatrchyan et al., Phys. Lett. B 718, 329-347 (2012). [arXiv:1208.2931]

196. ATLAS Collaboration Collaboration, G. Aad et al., JHEP 1212, 086 (2012). [arXiv:1210.4813]

197. B. Bhattacherjee, J. L. Evans, M. Ibe, S. Matsumoto, T. T. Yanagida [arXiv:1301.2336]

198. R. Franceschini, R. Mohapatra, JHEP 1304, 098 (2013). [arXiv: 1301.3637]

199. ALEPH Collaboration Collaboration, A. Heister et al., Eur. Phys. J. C31, 1-16 (2003). [hep-ex/0210014]

200. H.K. Dreiner, S. Grab, AIP Conf. Proc. 1200, 358 (2010). [arXiv: 0909.5407 [hep-ph]]

201. K. Desch, S. Fleischmann, P. Wienemann, H.K. Dreiner, S. Grab, Phys. Rev. D 83, 015013 (2011). [arXiv:1008.1580 [hep-ph]]

202. H.K. Dreiner, T. Stefaniak, Phys. Rev. D 86, 055010 (2012). [arXiv:1201.5014 [hep-ph]]

203. H.K. Dreiner, F. Staub, A. Vicente, W. Porod, Phys. Rev. D 86, 035021 (2012). [arXiv:1205.0557 [hep-ph]]
204. T. Kobayashi, Y. Nakai, R. Takahashi, JHEP 1001, 003 (2010). [arXiv:0910.3477 [hep-ph]]

205. N. Arkani-Hamed, S. Dimopoulos, JHEP 0506, 073 (2005). [hep-th/0405159]

206. N. Arkani-Hamed, S. Dimopoulos, G.F. Giudice, A. Romanino, Nucl. Phys. B 709, 3 (2005). [hep-ph/0409232]

207. G. F. Giudice and A. Romanino, Nucl. Phys. B 699, 65 (2004) [Erratum-ibid. B 706 (2005) 65] [hep-ph/0406088]

208. J. Jaeckel, V.V. Khoze, JHEP 1211, 115 (2012). [arXiv:1205.7091 [hep-ph]]

209. S. P. Martin, A supersymmetry primer, in Perspectives on supersymmetry II, ed. by G.L. Kane, pp. 1-153 [hep-ph/9709356]

210. J. Adam et al., MEG Collaboration. Phys. Rev. Lett. 107, 171801 (2011). [arXiv:1107.5547 [hep-ex]]

211. S. Abel, S. Khalil, O. Lebedev, Nucl. Phys. B 606, 151 (2001). [hep-ph/0103320]

212. S. Abel, O. Lebedev, JHEP 0601, 133 (2006). [hep-ph/0508135]

213. M. Raidal, A. van der Schaaf, I. Bigi, M.L. Mangano, Y.K. Semertzidis, S. Abel, S. Albino, S. Antusch et al., Eur. Phys. J. C 57, 13 (2008). [arXiv:0801.1826 [hep-ph]]

214. I. de Medeiros Varzielas, G. G. Ross [hep-ph/0612220]

215. J. J. Hudson, D. M. Kara, I. J. Smallman, B. E. Sauer, M. R. Tarbutt, E. A. Nature, 473, 493 (2011)

216. C. A. Baker, D. D. Doyle, P. Geltenbort, K. Green, M. G. D. van der Grinten, P. G. Harris, P. Iaydjiev, S. N. Ivanov et al., Phys. Rev. Lett. 97, 131801 (2006) [hep-ex/0602020]

217. W.C. Griffith, M.D. Swallows, T.H. Loftus, M.V. Romalis, B.R. Heckel, E.N. Fortson, Phys. Rev. Lett. 102, 101601 (2009)

218. G.G. Ross, L. Velasco-Sevilla, O. Vives, Nucl. Phys. B 692, 50 (2004). [hep-ph/0401064]

219. S. Antusch, S.F. King, M. Malinsky, JHEP 0806, 068 (2008). [arXiv:0708.1282 [hep-ph]]

220. S. Antusch, S.F. King, M. Malinsky, G.G. Ross, Phys. Lett. B 670, 383 (2009). [arXiv:0807.5047 [hep-ph]]

221. Z. Lalak, S. Pokorski, G.G. Ross, JHEP 1008, 129 (2010). [arXiv: 1006.2375 [hep-ph]]

222. F. Gabbiani, A. Masiero, Nucl. Phys. B 322, 235 (1989)

223. F. Gabbiani, E. Gabrielli, A. Masiero, L. Silvestrini, Nucl. Phys. B 477, 321 (1996). [hep-ph/9604387]

224. G. Isidori, Y. Nir, G. Perez, Annu. Rev. Nucl. Part. Sci. 60, 355 (2010). [arXiv:1002.0900 [hep-ph]]

225. G. Ross, M. Serna, Phys. Lett. B 664, 97 (2008). [arXiv:0704. 1248 [hep-ph]] 